\title{
Abhandlung
}

Stefan M. Maul*

\section{„Ninurta-Duft“ oder: Von den Vorsichts- maßnahmen, die ein mesopotamischer Heiler ergreifen sollte, bevor er einen erkrankten Patienten aufsuchte}

https://doi.org/10.1515/za-2018-0013

\begin{abstract}
Among the best preserved of the tablets found in the so-called "House of the Incantation Priest" in Assur numbers Tablet VAT 8803, already fired in antiquity, written by the healer Nabû-bēssun(u) in the early 7th century BCE. The accurate, archaising ductus of the script and the many triangular "firing holes" on the obverse, reverse, and side margins furnish this tablet, first published as KAR no. 31, with a striking physical appearance commensurate with its fundamental significance for ancient Near Eastern healing arts. In addition to instructions regarding the preparation and application of a salve which should provide the healer with the necessary sanitary protection when visiting invalids, the tablet also contains the wording of the concomitant exorcistic dicenda. A new edition of this text is here presented.
\end{abstract}

Olof Pedersén zum Geburtstag

Den bahnbrechenden Studien des Jubilars ist es $\mathrm{zu}$ danken, dass wir heute einen der bedeutendsten Tontafelfunde, die die deutschen Ausgräber von Assur zu Beginn des 20. Jh. machten, bis in Einzelheiten überschauen. ${ }^{1}$ In einem der Suchgräben, mit denen man das gesamte antike Stadtgebiet überzogen hatte, stießen Walter Andrae und seine Mitarbeiter im Sommer des Jahres 1908 auf die Reste eines Wohnhauses, das im ausgehenden siebten vorchristlichen Jahrhundert bei der Einnahme Assurs zerstört worden war. Die zusammenbrechenden Mauern hatten eine große, offenbar an mehreren Stellen aufbewahrte Sammlung von Tontafeln unter sich begraben. ${ }^{2}$ Es stellte sich bald heraus, dass die 1908 und 1910 nur teil-

1 Siehe Pedersén (1986, 41-76): N4. Private house with large library and archive of a family of exorcists (hC/D/E8I). Die hier verwendeten Abkürzungen richten sich nach dem Abkürzungsverzeichnis des Reallexikons der Assyriologie und Vorderasiatischen Archäologie (RlA). 2 Ein Plan der Hausreste mit Angabe der Fundstellen findet sich bei Pedersén (1986, 42 fig. 9). Siehe außerdem Miglus (1996, 236-241) mit den Plänen 41 und 132.

*Corresponding author: Stefan M. Maul, Universität Heidelberg, Heidelberg; Email: stefan.maul@ori.uni-heidelberg.de weise freigelegte Tafelsammlung gelehrten Heilern gehört hatte, deren Berufsbezeichnung, āšipu oder mašmaššu, von Assyriologen gewöhnlich mit „Exorzist“, „Beschwörer“ oder „Beschwörungspriester“ wiedergegeben wird.

Mit seinen grundlegenden Arbeiten lieferte Olof Pedersén den Schlüssel zu dem umfangreichen Schrifttum, das die Heiler von Assur zum Erlernen ihres Berufes benötigten. Die erstmals von Pedersén zusammengestellten Texte aus dem sog. Haus des Beschwörungspriesters lassen nämlich ein lebendiges Bild von der Tätigkeit der Heiler erstehen, die ihre Tafelsammlung zwar in ihrem privaten Wohnhaus aufbewahrt hatten, aber gleichwohl im Dienst des bedeutendsten Heiligtums des assyrischen Reiches, des Assur-Tempels, standen. Die insgesamt $631^{3}$ von Olof Pedersén in mühevoller Arbeit dem Tafelfund zugewiesenen Tontafeln und Tontafelfragmente zeigen, dass die Aufgabe der gelehrten Tafelbesitzer im wesentlichen darin bestand, jede Form von Unheil und Ungemach aufzuspüren, zu beseitigen und von dem König, seiner

3 Mittlerweile konnte Vf. zahlreiche weitere in Assur gefundene Stücke dem Tafelfund zuweisen, so dass nun mehr als 1300 Tafeln und Tafelbruchstücke aus dem sog. Haus des Beschwörungspriesters bekannt sind. 
Familie und auch von Land und Leuten fernzuhalten. ${ }^{4}$ Die Heiler hatten Krankheiten und Unpässlichkeiten aller Art zu bekämpfen, die Götter zu besänftigen und freundlich zu stimmen und außerdem aufmerksam auf jene Zeichen zu achten, die im Umfeld ihrer Schützlinge in Haus und Hof auftraten. Denn auch deren unheilvolle Folgen wollten sie schon im Vorfeld abwenden können.

\section{VAT 8803 (KAR 31)}

Unter den zahlreichen im sog. Haus des Beschwörungspriesters entdeckten Schriftstücken sticht die am 11. August 1908 gefundene Tontafel mit der Fundnummer Ass. $14240,{ }^{5}$ die heute im Berliner Vorderasiatischen Museum unter der Inventarnummer VAT 8803 aufbewahrt wird, hervor: nicht nur weil das im Hochkantformat $(12,3 \mathrm{~cm} \times 7,3 \mathrm{~cm} \times$ $1,7 \mathrm{~cm}$ ) beschriebene Objekt nahezu unbeschädigt blieb, sondern auch, weil es - wie nur wenige Stücke aus diesem Fundkomplex ${ }^{6}$ - bereits in der Antike gebrannt und mit zahlreichen, sehr auffälligen dreieckigen ,Brennlöchern ${ }^{67}$

$4 \mathrm{Zu}$ dem Tafelfund siehe auch Maul (2010).

5 Siehe Pedersén $(1986,70)$ zu N 4 Nr. 471.

6 Den Ausgrabungsberichten zufolge handelt es sich im einzelnen um (1) einen mittelassyrischen lexikalischen Text (malku = šarru II: VAT 9505 [Ass. 13936] = Pedersén 1986, 59 zu N 4 Nr. 1 sowie Hrůša 2010, 444 f. und Taf. 44), (2) eine mittelassyrische Götterliste (An = Anum V: VAT 9515 [Ass. 13944] = Pedersén 1986, 59 zu N 4 Nr. 2 sowie Litke 1998, 187-218 Text J), (3) die neuassyrische Abschrift der mittelassyrischen Beschreibung eines Rituals, durchzuführen im bìt ēqi von Kār-Tukultī-Ninurta (VAT 15421 [Ass. 14462] = Pedersén 1986, 70 zu N 4 Nr. 472 sowie Meinhold 2009, 349-354 und Taf. 26f.), (4) eine Tafel der Beschwörungsserie Maqlû (Maqlû VIII: A 480 [Ass. 14109] = Pedersén 1986, 70 zu N 4 Nr. 456 = Schwemer 2017, 41 und Pl. 116; spätneuassyrisch), (5) einen Kultkommentar (Marduk Ordeal und Kommentare: VAT 9538 [Ass. 13978] = Pedersén 1986, 69 zu N 4 Nr. 453 sowie Livingstone 1986, 236-243 und ders. 1989, 82-86 Text Nr. 34; spätneuassyrisch), (6) eine Tafel mit Eingeweideschauomina/Gallenblase (VAT 8611 [Ass. 17681 h] = Pedersén 1986, 71 zu N 4 Nr. 479 sowie Heeßel 2012, 126-128 und 405 Text Nr. 30; neuassyrisch), (7) einen astronomischen Text (MUL.APIN II: VAT 8619 [Ass. 17681 f] = Pedersén 1986, 71 zu N 4 Nr. 470 sowie Hunger 1989, Text NN; spätneuassyrisch), (8) eine Tafel mit Hemerologien für den Monat Tašrītu (VAT 8780 [Ass. 14047] = Pedersén 1986, 70 zu N 4 Nr. 455 sowie Casaburi 2000 und ferner Livingstone 2014, 177-192 Text B; frühneuassyrisch) und um zwei Königsinschriften: (9) die spätneuassyrische Kopie einer Inschrift des Aššur-uballiț I. (VA 5707 [Ass. 13963] = Pedersén 1986, 69 zu N 4 Nr. 451 sowie Grayson 1987, 109f.) sowie (10) eine Beschreibung des achten Feldzugs Sargons II. (AO 5372 [+] VAT 8634 + VAT 8698 + VAT 8749 [Ass. $17681 \mathrm{a}+\mathrm{b}+\mathrm{c}+\mathrm{d}+\mathrm{g}+$ ] = Pedersén 1986, 71 zu N 4 Nr. 477). Hinzu kommt (11) der hier studierte Text.

7 Die Löcher wurden mit einem dreikantigen Gegenstand auf die Seitenränder und an freigebliebenen Stellen der Schriftzeilen auf Vorder- und Rückseite der Tafel in den noch plastischen Ton gestochen. versehen wurde, die sich sonst auf keiner weiteren Tontafel aus Assur finden. ${ }^{8}$ Darüber hinaus zeichnet sich die Tafel durch eine auffällig saubere, klare neuassyrische Beschriftung aus. Bei genauerer Betrachtung unterscheidet sich der Schriftduktus deutlich von der spätneuassyrischen Schrift, welche typisch für die meisten Tontafeln aus dem sog. Haus des Beschwörungspriesters ist. Die Proportionen der Keilschriftzeichen in VAT 8803 erinnern noch sehr an frühneuassyrische Schriftzeugnisse, und manche Keilschriftzeichen weisen altertümliche Formen auf, die man im Fach bislang ausschließlich für ,mittelassyrisch“ hält (siehe Borger ${ }^{21981, ~ 1-35): ~ s o ~ d a s ~ R U ~ m i t ~ v i e r ~}$ stehenden Keilen am Ende'; das šu mit insgesamt fünf waagerechten Keilen ${ }^{10}$; das TA, das mit insgesamt fünf liegenden Keilen beginnt ${ }^{11}$; die Zeichen $\mathrm{TU}^{12}$ und $\mathrm{LI}^{13}$, deren vorderer Abschnitt von 2+4+4 liegenden Keilen gebildet ist; das RA, dessen erster Teil aus 1+3 waagerechten Keilen besteht, ${ }^{14}$ und schließlich die komplexe Zeichenform des GIG, die mit einem Winkelhaken beginnt, auf den 5+4 liegende Keile folgen. ${ }^{15}$

Der Tafelschreiber verwendete hierfür möglicherweise den rückseitigen Abschnitt seines Schreibgriffels oder eines für Tafelherstellung und -aufbereitung verwendeten Glättinstrumentes. Die durch das Einstechen entstehenden gleichseitigen Dreiecke haben eine Seitenlänge von etwa 2,5 mm. Die 51 auf der Vorderseite und die 24 auf der Rückseite befindlichen ,Brennlöcher‘ (nicht alle sind in der Autographie von E. Ebeling, KAR Nr. 31, wiedergegeben) - wurden so angebracht, dass die gleichseitigen Dreiecke jeweils mit einer Spitze auf der Standlinie einer Zeile stehen. Nur in drei Fällen (VAT 8803, Vs. 5 und 10 [dort das zweite und dritte ,Brennloch']) wurden gegeneinander versetzt zwei Dreiecke eingestochen, so dass dabei ein davidsternartiger Eindruck entstand. Auf dem oberen und auf dem unteren Tafelrand befinden sich in regelmäßigen Abständen jeweils 6, auf den Seitenrändern jeweils 9 dreieckige Löcher. Zu der Tafel, den darauf befindlichen ,Brennlöchern' und Überlegungen zur Rekonstruktion des Instruments, mit dem die ,Brennlöcher‘ gestochen wurden, siehe auch Marzahn (2017).

8 Aus den königlichen Bibliotheken zu Ninive ist mir zumindest eine Tontafel bekannt, die ebenfalls dreieckige ,Brennlöcher` aufweist (K 2875 + K 5838 + K 9930; Balaĝ, babylonische Schrift; siehe das Photo CDLI P238192).

9 VAT 8803, Vs. 4, 16, 31, Rs. 2, 15 (in: pàd), 20.

10 VAT 8803, Vs. 5, 6, 14, 22, 28, Rs. 5, 6, 16, 25, 28.

11 VAT 8803, Vs. 7, 19, 21, 31, Rs. 6, 9, 10, 11, 12, 17, 20.

12 VAT 8803, Vs. 8, 12, Rs. 3, 8 (Diese komplexe Form des Zeichens TU ist weder in Borger 21981 noch in Labat 61988 verzeichnet).

13 VAT 8803, Vs. 18, 36 (Diese komplexe Form des Zeichens LI ist weder in Borger 21981 noch in Labat ${ }^{6} 1988$ verzeichnet).

14 VAT 8803, Vs. 16, 21, 23, 31, 33, Rs. 12, 19, 22, 24.

15 VAT 8803, Rs. 22, 23 (Diese Zeichenform ist weder in Borger 21981 noch in Labat 61988 verzeichnet). 


\section{Nabû-bēssun(u), der Schreiber der Tafel}

Da im Kolophon, mit dem die Tafel versehen ist (Rs. 24-28), der Name des Schreibers Nabû-bēssun(u) genannt ist, kann VAT 8803 und damit auch der in VAT 8803 vorliegende Schriftduktus recht genau datiert werden. Denn wir wissen, dass Nabû-bēssun(u) in Assur wohl in den 80er Jahren des siebten Jahrhunderts als „Beschwörer des Assur-Tempels“ eine führende Stellung als Heiler einnahm. ${ }^{16}$ Dank mehrerer uns bekannt gewordener Tafelunterschriften von seiner Hand lässt sich die Karriere dieses Ahnherrn der Beschwörer, die in der spätneuassyrischen Zeit den größten Teil der Tontafeln aus dem sog. Haus des Beschwörungspriesters schrieben, gut verfolgen. ${ }^{17}$ An der Wende vom 8. zum 7. Jh. dürfte Nabû-bēssun(u) als ,junger Assistent“ (šamallû șeḩru) im Hause seines Vaters Bābašuma-ibni tätig gewesen sein. Im Verlauf des ersten Drittels des 7. Jhs. stieg er zunächst zum „jungen Beschwörer“ $(\text { mašmaššu șehru })^{18}$, dann zum „Beschwörer von Assur“

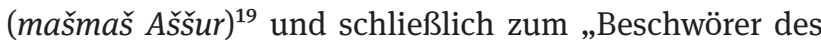
Assur-Tempels“ (mašmaš bït Aššur) ${ }^{20}$ auf.

Im Kolophon der Tafel VAT 8803 hat Nabû-bēssun(u) (so wie auch in dem Kolophon der Tafel EHE 338 ${ }^{21}$ ) auf die Angabe eines Titels verzichtet, der Auskunft über seine damalige Position oder Qualifikation hätte geben können. So können wir nur darüber mutmaßen, dass die hier besprochene Tafel - so wie dies auch für viele weitere Tafeln aus der sog. Bibliothek aus dem Haus des Beschwörungspriesters gilt ${ }^{22}$ - von dem noch jungen Gelehrten zum Nachweis seines Könnens angefertigt und geschrieben wurde und vielleicht gerade aus diesem Grund $\mathrm{zu}$ den wenigen Tafeln von der Hand des Nabû-bēssun(u) gehört, die in der Tafelsammlung aus dem sog. Haus des Beschwörungspriesters aufbewahrt wurden und die Zeiten überdauerten.

In dem sehr breiten Feld der ,Heil- und Beschwörungskunst' (āšipūtu) kommt dem in VAT 8803 überlieferten Text, der uns auch aus zwei bruchstückhaft erhalte-

16 Siehe Maul $(2010,205)$.

17 Siehe Maul (2010, 209).

18 VAT 14214 (Ass. $17722 \mathrm{fn}=$ KAL 4 Nr. 6): Rs. $3^{\prime}$.

19 VAT 13652 (Ass. 17721 z = LKA 109 = Maul 1994, 467-483; siehe Hunger 1968, 68 Nr. 194 und Pedersén 1986, 71 zu Nr. 499): Rs. 15'.

20 VAT 8960 (Ass. 13955 da = KAR 64 = Maul 1994, 312-322; siehe Hunger 1968, 67 f. Nr. 193 und Pedersén 1986, 61 zu Nr. 91): Rs. 24.

21 EHE 338 (ohne Fundnummer = Scheil 1921, 27-29 Text Nr. 18 = Maul 1994, 421-431; siehe Hunger 1968, 68 Nr. 195; Durand 1982, 24 und Pedersén 1986, 75 zu Nr. 624).

22 Siehe Maul (2010, 215). nen, unveröffentlichten neuassyrischen Abschriften aus den königlichen Bibliotheken $\mathrm{zu}$ Ninive bekannt ist, ${ }^{23}$ eine zentrale Stellung zu. Das in VAT 8803 beschriebene Verfahren, dem sich ein „Beschwörer“ zu seinem eigenen Schutz zu unterziehen hatte, ist nämlich - selbst im wörtlichsten Sinn - als Anfang jeder ,Heil- und Beschwörungskunst' zu betrachten. Denn ein mesopotamischer Heiler hatte jeden Morgen aufs neue, immer, bevor er sich auf den Weg begab, um Patienten aufzusuchen und gegen Unheil und Leiden vorzugehen, mit der in VAT 8803 geschilderten Selbstbehandlung sicherzustellen, dass er vor jeglichem Übel gefeit blieb und dieses nicht an unbeteiligte Dritte weitergab, auch wenn er bei seiner Arbeit immer wieder mit Beschwerden aller Art, mit gefährlichen Krankheiten und Tod in Berührung kam.

Es ist durchaus denkbar, dass mit der ansprechend gestalteten, durch Brand gehärteten und so dauerhaft gemachten Tafel VAT 8803 eine Art ,Meisterstück des jungen Heilers vorliegt, das dieser - vielleicht zum Anlass des Übergangs von einer Ausbildungsstufe in die nächste ${ }^{24}$ - vorgelegt hatte. In der heilkundlichen Ausbildung im Assur des 7. Jhs. stellte der Schritt vom Assistenten (šamallû) zum Heiler (mašmaššu) einen besonders großen Einschnitt dar. Denn der Bereich der Heilkunst, der sich mit Herstellung und Verabreichung von Medikamenten befasste, war allein den Fortgeschrittenen vorbehalten, die mit dem Recht, den Titel mašmaššu zu führen, offenbar auch eine Art Approbation erwarben. ${ }^{25}$ $\mathrm{Zu}$ einem solchen, möglicherweise feierlich gerahmten Anlass könnte auch VAT 8803 geschrieben worden sein.

\section{Unheil, Krankheit und die Gefahr der Übertragung}

Ein altbabylonischer Brief aus Mari ${ }^{26}$ stellt eindrucksvoll unter Beweis, dass schon im 18. Jahrhundert mesopotamischen Heilern vollkommen bewusst war, dass Krankheiten durch direkten oder indirekten Kontakt mit ihren

23 K 9836 + K 10338 + K 20638 und K 10565, Vs. Hierzu siehe unten. $24 \mathrm{Zu}$ diesen Ausbildungs- und Qualifikationsstufen siehe Maul (2010, 208-211).

25 Siehe Maul $(2010,216)$.

26 Zu dem Brief ARM 10, Nr. 129 (vgl. auch ARM 10, Nr. 14 und Nr. 130) siehe Durand (2000, 343-347) mit einer neuen Übersetzung, mit Literaturhinweisen und Kollationsergebnissen. $\mathrm{Zu}$ dem Text siehe auch Neufeld (1986). 


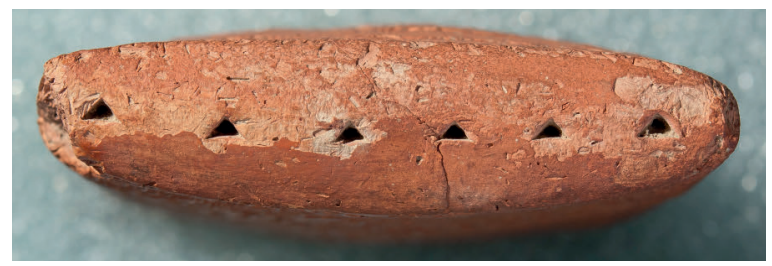

VAT 8803 , oberer Rand

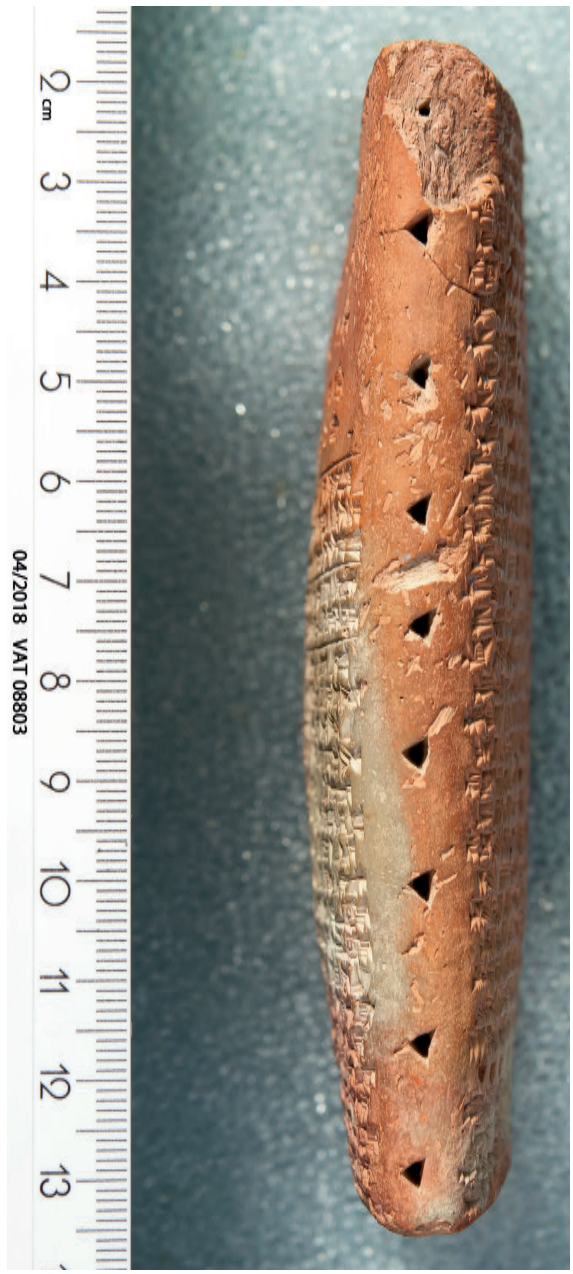

VAT 8803 , linker Rand

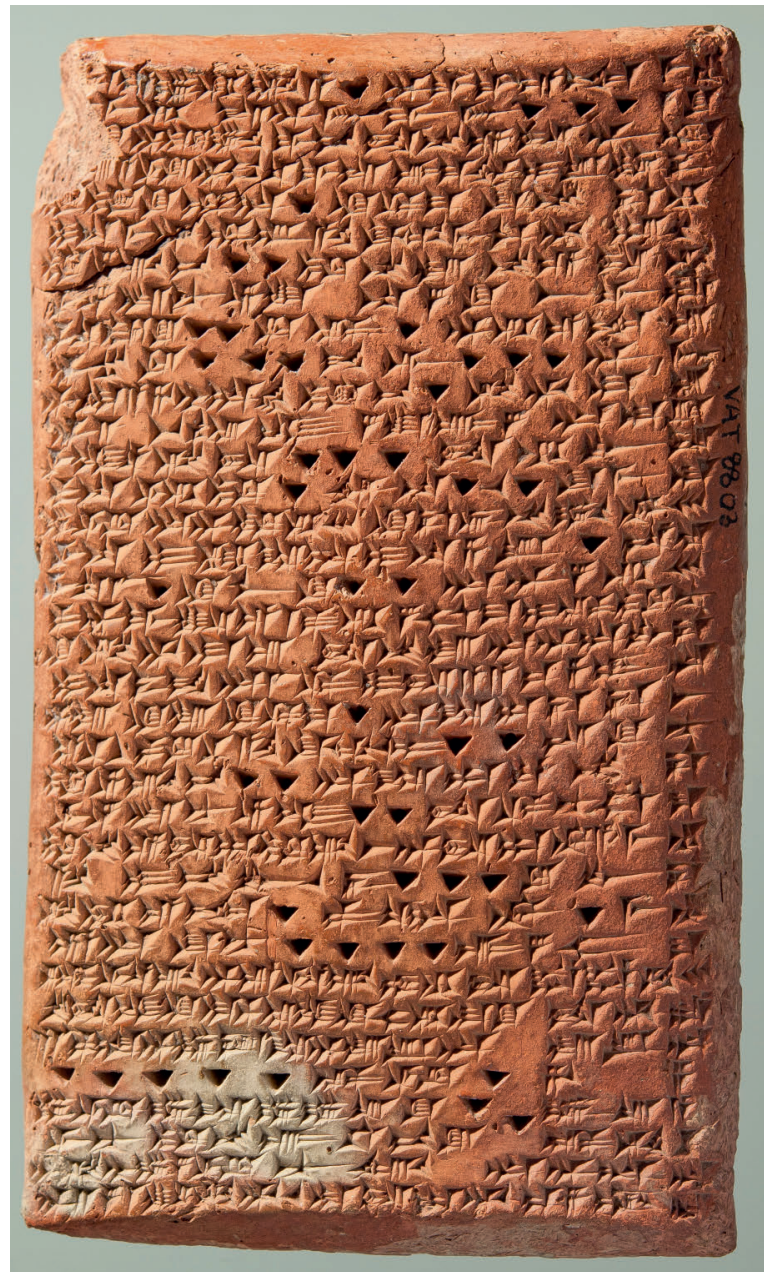

VAT 8803, Vorderseite

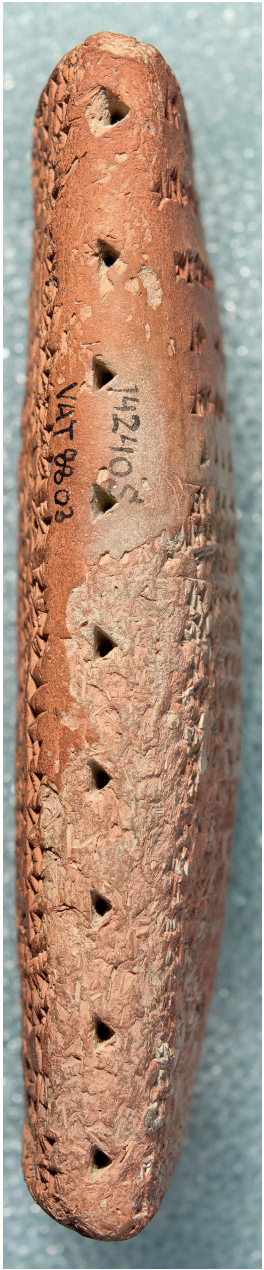

VAT 8803, rechter Rand
Patienten übertragen werden können. ${ }^{27}$ In dem an die Königin gerichteten Brief wird nämlich die strikte Anweisung erteilt, eine Dame, die im Palast von Mari lebte und an einem simmum genannten Leiden erkrankt war, von allen anderen Menschen fernzuhalten und außerdem dafür zu sorgen, dass Dritte nicht in Berührung mit dem

$27 \mathrm{Zu}$ mesopotamischen Konzepten, die unserer Vorstellung von Ansteckung weitgehend entsprechen, siehe Farber (2004), Couto (2007) sowie Attinger (2008, 45 und 63f.). von ihr benutzten Geschirr oder ihren Möbeln kamen, da „diese Art von simmum leicht übertragen werden“ könne (simmum šū muštahhiz). In dem Schrifttum der Heiler des ersten Jahrtausends ist ein Verfahren genannt, das marșa ana esēri („Um einen Kranken abzusondern“) hieß und wohl Anweisungen für entsprechende seuchenhygienische Maßnahmen enthielt. ${ }^{28}$ Ein Brief aus dem siebten 


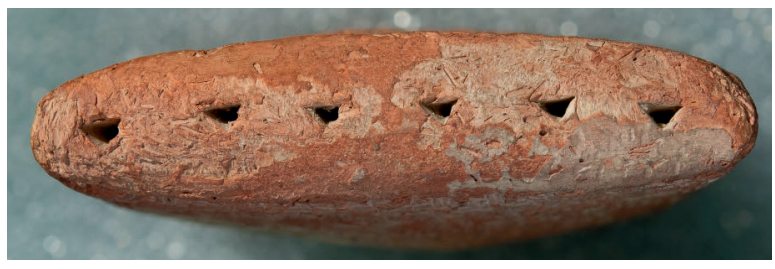

VAT 8803, unterer Rand

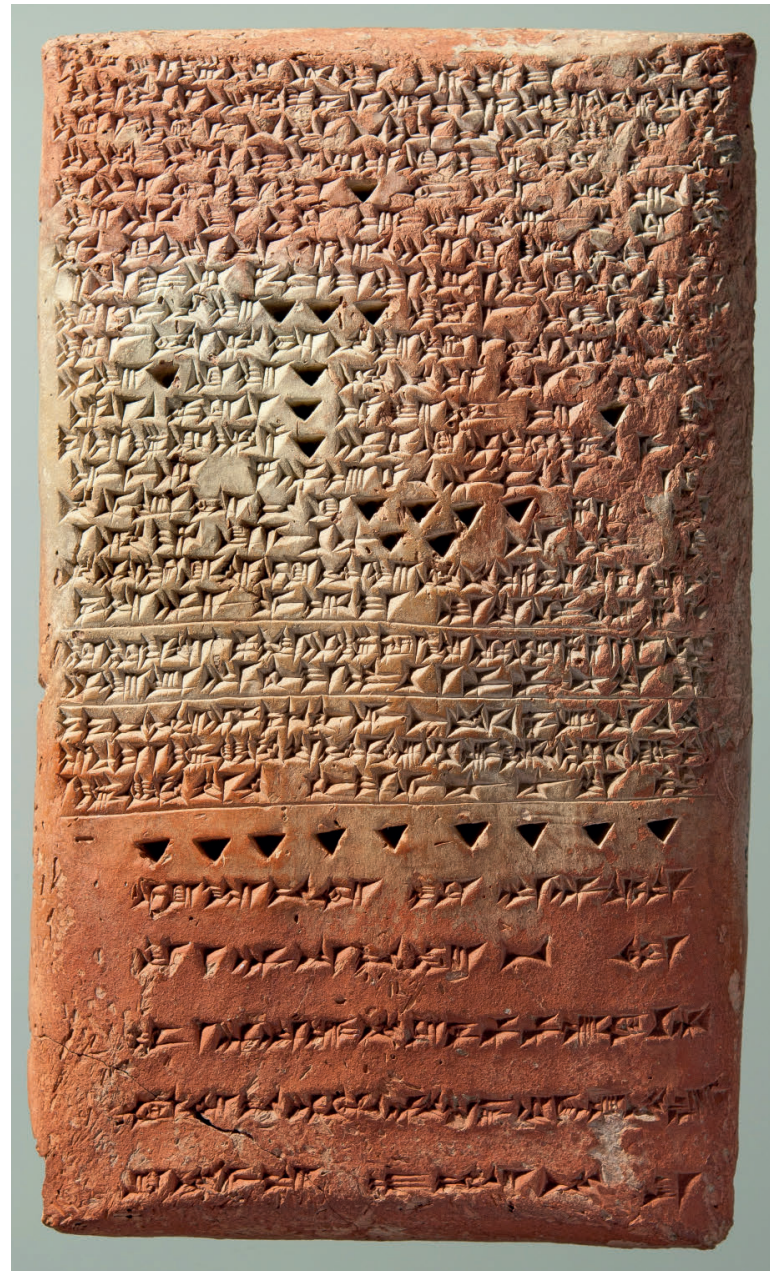

VAT 8803, Rückseite

Jahrhundert ${ }^{29}$, den der Beschwörer Adad-šuma-ușur ${ }^{30}$ an König Asarhaddon sandte, verdeutlicht, welche Vorsicht kluge Heiler im Umgang mit ihren Patienten walten ließen, um die Gefahr der Ansteckung zu mindern. Der zu einem kranken Prinzen beorderte Heiler ließ den König nämlich in seinem Schreiben wissen, dass er den Königssohn besser nicht sofort, sondern erst am folgen-

29 Parpola 1993, 175 Text Nr. 222.

$30 \mathrm{Zu}$ Adad-šuma-ușur siehe Parpola (1983, 28). den Tag aufsuchen werde, da er zuvor einen „sehr, sehr kranken“ Jungen behandelt hatte. Da er befürchtete, er könne die Krankheit des leidenden Jungen an den Prinzen weitergeben, wollte er diesem vorerst lieber fern bleiben und zog es vor, Kollegen zu schicken, um ihn zu behandeln.

Die Erfahrung, dass direkter oder indirekter Kontakt mit einem Infizierten zu einer Erkrankung führen kann, spiegelt sich auch in litaneiartigen Aufzählungen, die sich in Beschwörungen aus dem ersten Jahrtausend finden und den Grund für das Erkranken einer Person zu benennen suchen. Obgleich die zu therapierende Erkrankung hier grundsätzlich als Folge eines Urteils betrachtet wurde, das Götter in ihrem Ärger über einen Missetäter gesprochen hatten, sah man doch in der Übertragung eines Übels von einem Menschen auf den anderen oder aber im Kontakt mit unreinen Stoffen die Weise, wie das zuvor gefällte göttliche Urteil an den Schuldigen herangeführt wurde, um sich zu materialisieren. Zwei Passagen aus den sog. lipšur-Litaneien veranschaulichen dies besonders gut:

Mag N.N., der Sohn des N.N., ${ }^{31}$ gefehlt haben, er sei davon entbunden, es sei ihm getilgt! Mag er gefrevelt, mag er sich vergangen haben, ditto (d.h.: er sei davon entbunden, es sei ihm getilgt!). Mag er Gewalttätigkeiten begangen haben, ditto! Mag er eine Schlägerei angezettelt haben, ditto! Mag er in Unwissenheit das gegessen haben, was seinem Gott zuwider ist, ditto! Mag er sich an die entu-Priesterin seines Gottes herangemacht haben, ditto! Mag er die Ehefrau seines Genossen belästigt haben, ditto! Mag er seinem Gott Ungutes angetan haben, ditto! Mag er mit jemandem gesprochen haben, der unter einem Bann steht, ditto! Mag er das Brot einer Person gegessen haben, die unter einem Bann steht; mag er das Wasser einer Person getrunken haben, die unter einem Bann steht, ditto! Mag er das getrunken haben, was eine Person, die unter einem Bann steht, übrig gelassen hatte; mag er mit demjenigen, der eine Schuldenlast auf sich geladen hat, gesprochen haben, ditto! Mag er das Brot einer Person gegessen haben, die eine Schuldenlast auf sich geladen hat; mag er das Wasser einer Person getrunken haben, die eine Schuldenlast auf sich geladen hat; mag er Fürsprache eingelegt haben für eine Person, die eine Schuldenlast auf sich geladen hat, ditto! ${ }^{32}$

Als ich eine Straße entlangging, mag mich jemand, der unter einem Bann steht, berührt haben. Als ich einen Platz überquerte, mag ich in das zum Waschen benutzte Wasser, in nicht Aufgewischtes getreten sein. Abgeschnittene Fingernägel, aus den Achselhöhlen ausrasierte Haare, ein löchriger Schuh, ein

31 Ein Textvertreter statt dessen: „N.N., der Sohn seines Gottes“. 32 Aus einer lipšur-Litanei, die ihren ,Sitz im Leben` in einer Therapie hatte, die nam-érim-búr-ru-da (,Bannlösung“) genannt wurde (siehe Reiner 1956, 136 f.: 81-88). 
durchtrennter Gürtel, ein ,Zaubersack ${ }^{633}$, Aussatz, ein mehru ${ }^{34}$ der Menschheit - auf all diese Dinge mag ich zugegangen sein. Seien sie doch gelöst von mir, sei ich doch davon entbunden! ${ }^{35}$

Daher erstaunt es wenig, dass die Heiler damit rechneten, dass bei Krankenbesuchen die Leiden, die es sich bei ihren Patienten „bequem gemacht“36 hatten, sich ihnen selbst an die Fersen heften und auch in ihr Haus und ihre Wohnräume eindringen könnten. ${ }^{37}$ Ihrem Weltbild entsprechend sahen sie in den ihnen in ihren Patienten entgegentretenden Krankheiten intelligente, handelnde und willensbegabte bösartige Gegenüber, die sich aber als benennbar, ansprechbar und - wenn man über einschlägige Kenntnisse und die hierfür notwendige Macht verfügte - auch als beeinflussbar erwiesen. So versuchten die Beschwörer, nicht allein Gewalt über die Krankheiten und Übel verkörpernden Wesen zu erlangen, um ihre Patienten von deren Zugriff zu befreien, sondern auch, um sie von der eigenen Person und Familie sowie von Haus und Hof fernhalten zu können.

\section{Das in VAT 8803 beschriebene Verfahren der Feiung des Heilers vor Unheil und Krankheit}

Das Manuskript des Nabû-bēssun(u) enthält in kurzer Form das gesamte Credo der mesopotamischen Heiler. In wenigen Zeilen sind in dem zu rezitierenden sumerischen Text die elementaren Wesenszüge der altorientalischen „Beschwörungskunst“ (āšipūtu) dargelegt:

Der „Beschwörer“ versteht sich als Sendbote der großen Götter (Z. 1), versehen mit dem Auftrag, menschliches Leid $\mathrm{zu}$ lindern. Hierfür ist er ausgestattet mit dem ihm offenbarten Wissen und der Kunst der sich des Menschen in seiner Not erbarmenden Götter der Weisheit (Z. 2). Wenn der „Beschwörer“ als Heiler in göttlichem Auftrag handelt, verkörpert er den Gottessohn

33 Ein Ledersäckchen, das, um Schadenzauber zu bewirken, mit magisch kontaminierten ,Unheilsstoffen' gefüllt und mit einem Menschen in Kontakt gebracht wurde.

34 mehru bezeichnet hier einen mit magisch kontaminierten Stoffen versehenen ,Unheilsträger‘. Der Zusatz „der Menschheit“ will besagen, dass das mehru von einem (menschlichen) ,Zauberer' mit der Absicht, durch Kontakt Schaden zu bewirken, gefertigt wurde.

35 Aus einer lipšur-Litanei, die ihren ,Sitz im Leben' in einer Therapie hatte, die nam-érim-búr-ru-da („Bannlösung“) genannt wurde (siehe Reiner 1956, 142f.: 41'-47').

36 Siehe BAM 8, 91: 6 (von den Dämonen gesagt: sa ni ĝin-nabi(-e) nu(-un)-zu ki tu-ra ba(-an)-nú = naphar šer'ānišu ilmadūma ašar marși irtabșū) und ferner 103: 63.

37 Siehe z. B. BAM 8, 106: 73-76.
Asalluhi (Z. 2), der - vom Vater instruiert - zu den Menschen gesandt ist. Asalluhi selbst ist es, der durch den Heiler spricht. ${ }^{38}$ Die Macht, über Unheil zu gebieten und Heil durch Handauflegung einkehren zu lassen (Z. 3), kommt über den Sohn Asalluhi von Enki/Ea, dem Vater. Sie liegt zuallererst in dem befehlenden Gotteswort (Z. 3-4), dem sich die dunklen, dem Menschen Schaden zufügenden Mächte letzten Endes immer zu beugen haben.

Der Heiler glaubt, unbeirrbar dem schlimmsten Übel und so auch ansteckenden Krankheiten entgegentreten zu können, da er sich behütet sieht von einer göttlichen Macht, die Böses abwehrt und gleichzeitig Leben spendet. In seinen Augen fügt sich diese Macht aus zwei Teilen, aus einer männlichen und einer weiblichen Kraft, die beide als eigene göttliche Personen gedacht sind (Z. 5-6). Der (männliche) ,Schutzengel' (da la d = šêdu) und der weibliche, gute Geist' ( ${ }^{\mathrm{d}}$ l a m m a = lamassu), die der Weltsicht der Heiler zufolge jeden Menschen, der sich als „Gotteskind“ (lú- $\mathrm{u}_{18}-\mathrm{lu}$ dumu dîg ir-a-na = amēlu mār ilišu $)^{39}$ bezeichnet, umgeben, ${ }^{40}$ sind für den Heiler ein Götterpaar, in dem man mit gewissem Recht die ,Patrone' der „Beschwörungskunst“" sehen darf: Uta’ulu, eine Erscheinungsform des kriegerischen Heldengottes, der dereinst durch seinen Kampf und Sieg die Welt vor den dunkeln Mächten bewahrte und so die Schöpfung ermöglichte, und Ningirima, ${ }^{41}$ die eng mit dem heilenden Wasser verbundene Göttin des beschwörenden Wortes. ${ }^{42}$ Der wohl schon in altbabylonischer Zeit mit Ninurta gleichgesetzte $\mathrm{Uta}^{\prime} \mathrm{ulu}^{43}$ steht für eine offensive Energie, die durch aktive Verteidigung das Böse vertreibt und drohend fernhält, während in Ningirima die heilbringenden Kräfte Gestalt gewinnen, die mit Zuwendung und Fürsorge durch das elementare Heilmittel ,Wasser ${ }^{`}$ sowie die Macht von Gotteswort und Heilkunst zum Leben zurückführen. Beide gemeinsam stehen in allen Richtungen den bösen Kräften, die den Heiler umgeben könnten, entgegen, um sie zu vertreiben (Z. 7 und Z. 11). Vor ihnen müssen die Unheilswesen weichen, denn dort, wo Uta'ulu und Ningirima zugegen sind, obsiegt das Leben (Z. 10).

38 Siehe den Kommentar zu Z. 2.

39 Siehe z. B. BAM 8, 416: 97 und passim in dem Schrifttum der „Beschwörer" (äšipūtu).

40 Hierzu siehe unten den Kommentar zu Z. 15.

41 Siehe Krebernik (1998) und Lambert (2013, 427-436).

42 An anderer Stelle wird Enki selbst angerufen, dem „Beschwörer“ ,Schutzengel' und ,guter Geist' zu sein (BAM 8, 130 f.: 191f.).

43 Siehe Maul (1991, 314: 8 und 319f.) sowie Streck (1998, 519). Als Schützer des Heilers sind im Schrifttum der „Beschwörer“ auch Ninurta (BAM 8, 112: 110; 206: 148) und der mit ihm gleichgesetzte Ninĝirsu (BAM 8, 94: 17; 106: 71) genannt. 
Die Tätigkeit des „Beschwörers“, das Kurieren mit Wort und Tat, gilt dem Heiler als Auftrag, uranfängliches göttliches Handeln zum Heil der Menschen zu wiederholen (Z. 12f.). In diesem Sinne sieht man in jedem Auftritt des „Beschwörers“ ein im Hier und Jetzt erfahrbares Abbild göttlicher Heilstat. Der von einem „Beschwörer“ bewirkte Heilerfolg gerät so nahezu wie von selbst zum Gotteslob (Z. 14) und fordert dieses bei den Zeugen heraus. ${ }^{44} \mathrm{Nich}$ zuletzt aus diesem Grund darf der Heiler, der durch sein Tun die Ehre der Götter mehrt, auf den Segen der höheren Mächte hoffen (Z. 15).

Er verfügt so über die gottgegebene Macht, die Geistwesen des Kranken und Bösen (Z. 16-19) aufzustören, anzusprechen und ihnen den Befehl zu erteilen zurückzuweichen (Z. 20-22), ohne dass sie zum Gegenangriff ausholen und den von den Göttern gewährten Schutzraum verletzen können. Denn dieser schließt den Heiler rundum ein wie in einem Ei und macht ihn unangreifbar (Z. 24f.). Allein der Klang des heiligen Namens von Enki und Asalluhi, des göttlichen Vaters und seines Sohnes (Z. 26 f.), zwingt alle Übel, vor dem „Beschwörer“ zurückzuweichen. Die den Übeln unerträgliche, stets den „Beschwörer“ begleitende Lebenskraft des Heldengottes Uta'ulu ist es, die dem Heiler dauerhaften Schutz gewähren soll (Z. 28).

Nur durch die Handlungsanweisung, die dem in sumerischer Sprache zu rezitierenden (und lediglich für den Lernenden ins Akkadische übertragenen) Exorzismus beigegeben wurde (Z. 31-33), wird klar, dass die Feiung des Heilers nicht allein religiöser Natur war. Sie besaß auch eine ganz handfeste materielle Grundlage. Der Heiler sollte sich nämlich zum eigenen Schutz am ganzen Körper (ištēniš) mit einer Salbe einreiben, die er auf der Grundlage von Honig und Butterschmalz fertigen und mit einem pflanzlichen Arzneimittel versetzen sollte, das die Babylonier nikiptu (bzw. niqiptu) nannten..$^{45}$

R. C. Thompson vermutete in nik/qiptu die baumhoch werdende ${ }^{46}$ Wolfsmilchart Euphorbia Antiquorum L ${ }^{47}$ Bei der Herstellung der schützenden Salbe ${ }^{48}$ kamen nebenein-

44 Hierzu siehe auch Mayer (1976, 310-347).

45 Bereits in altbabylonischer Zeit sind möglicherweise recht ähnliche Selbstschutzmaßnahmen des Heilers bezeugt (Cavigneaux 1999, 264-270), die ebenfalls mit einer Salbung einhergehen.

46 Aus Knudtzon (1915), Text Nr. 41: 43 ergibt sich, dass nik/qiptu in Gestalt von „großen Bäumen“ verhandelt werden konnte. Die Zugehörigkeit zu den „Hölzern“ läßt sich bereits aus sehr frühen Textzeugnissen erschließen (Brunke/Sallaberger 2010, 50 zu Nr. 12).

47 Thompson 1949, 364-367.

48 nik/qiptu wurde als Bestandteil von Salben außer in dem hier beschriebenen Zusammenhang auch zum Schutz gegen den Zugriff des Totengeistes (siehe Scurlock 2006, 303 Nr. 88-89; 323 Nr. 104; ander zwei unterschiedliche Spielarten des Arzneimittels zur Anwendung: eine von den mesopotamischen Heilern als männlich und eine als weiblich bezeichnete Form. Aus einem medizinischen Kommentartext, der im vierten vorchristlichen Jahrhundert in Uruk niedergeschrieben wurde, erfahren wir, dass „männliches nik/qiptu wie Tamariskenrinde, kompakt und rot“, aber „weibliches nik/qiptu wie Tamariskenrinde, ganz fein und gelblich“49 sei. Möglicherweise handelt es sich bei männlichem und weiblichem nik/qiptu also um Rinden- oder Holzstücke der nik/qiptu-Pflanze, die sich in Konsistenz und Erscheinungsbild deutlich unterscheiden. Genaueres lässt sich derzeit wohl noch nicht sagen. Aufschlussreich hingegen ist die zur Schreibung des Pflanzennamens nik/qiptu nicht seltene verwendete keilschriftliche Graphie šIM. ${ }^{\mathrm{d}} \mathrm{MAŠ}$ mit der Variante ŠIM. ${ }^{d}$ NIN.URTA, die im Sumerischen mit der Aussprache ligidba ${ }^{50}$ belegt war (siehe CAD N/2, 222 s. v. nikiptu A), aber unter ideographischen Gesichtspunkten betrachtet „Ninurta-Duft“ bedeutet. Hieraus muss man folgern, dass nik/qiptu einen starken Geruch besa $\beta^{51}$ und gerade hierin - zumindest in dem hier besprochenen Zusammenhang - die Wirkkraft der Pflanze lag.52

334-336 Nr. 114a; 337 f. Nr. 114b; 348f. Nr. 116; 439 Nr. 175; 489 Nr. 205; 498 Nr. 213; 500 Nr. 214; 501 Nr. 215; 607 Nr. 290; 609 Nr. 292; 611 Nr. 294; 613 Nr. 296; 616 Nr. 299; 617 Nr. 300) und gegen Schadenzauber (Abusch/Schwemer 2011, 242: 10-12) eingesetzt. Das Rezept für eine nik/qiptu enthaltende Salbe, die für einen erkrankten assyrischen Prinzen hergestellt werden sollte, nennt die mittelassyrische Urkunde VAT 10044 (MARV 2, Nr. 28 = Jakob 2003, 533).

49 BRM 4, 32: 11f. (siehe Thompson 1924 und Geller 2010, 168-173): ŠIM. ${ }^{\mathrm{d} M A S ̌ ~ N I T A ~ k i-m a ~ q u-l e p-t u ́ ~ s ̂ i s ̌ s ̌ I N I G ~ k a-s ̦ a r ~ u ~} \mathrm{SA}_{5}$ ni-kip-tú MUNUS ki-ma qu-lep-tú ŝiššinIG / raq-qa-qu u a-ra-qu.

50 Ältere Schreibungen sind bei Brunke/Sallaberger (2010, 50)

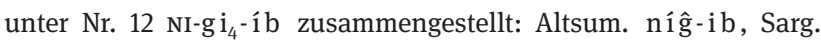
ĝešNI-ki-íb, Gudea-zeitlich nin-ki-ib-tum oder nin-ki-ibíb, UrIII-zeitlich dnin-ib-tum bzw. šem NI-ki-íb, Umma NI-gi $i_{4}-i ́ b$. Dabei ist „offensichtlich /nigibt-/ > /ligibd-/ > /ligidb-/“ geworden. Die Verbindung zu Ninurta kommt offenbar über eine Umdeutung der Schreibung nî $\hat{g} / \mathrm{nin}-\mathrm{ib}$ zustande.

51 Schon sehr frühe Texte zeigen, dass ligi d b a wegen seiner Duftqualität geschätzt wurde (siehe Brunke/Sallaberger 2010, 50). Der hohe Preis, der für lig id ba gezahlt wurde (ebd., 72), spricht dafür, dass das Holz importiert werden musste.

52 Zu der Verwendung von nik/qiptu als Simplicium oder im Verbund mit weiteren Heilmitteln siehe Thompson (1949, 364 f.). Als Heilmittel kam nik/qiptu auf vielfältige Weise zum Einsatz. So wurde es nicht nur für Salben verwendet, sondern mit Kräutern, Steinen und anderen Materialien in einem Lederbeutel um den Hals getragen, etwa zum Schutz gegen das Wirken eines Totengeistes (Scurlock 2006, 237 Nr. 31; siehe auch ebd., 429 Nr. 167; 488 Nr. 204: 1; 489 Nr. 205: 3; 491 Nr. 206: 4; 494 Nr. 209; 495 Nr. 210; 500 Nr. 214; 548 Nr. 235: 5; 553 Nr. 238; 556 Nr. 241; 557 Nr. 242: 1) oder gegen Schadenzauber und anderes Unheil (Abusch/Schwemer 2011, 114, 5.2: 1-3; Farber 1977, 244: 89'-92'). Mit weiteren Pflanzen auf eine Kette aufgereiht sollte es 
Aus der Sicht der Heiler materialisierte sich demnach die Kraft des kriegerischen, rächenden Heldengottes, der dem „Beschwörer“ als ,Schutzengel` zur Seite geht, im Olfaktorischen. Man darf wohl annehmen, dass der die bösen Geister in die Flucht schlagende „Ninurta-Duft“ kein lieblicher war, sondern vielmehr ein recht strenger, vielleicht sogar ein übelriechender. ${ }^{53}$ In Uruanna, dem ,Pharmakologischen Handbuch ${ }^{64}$ der mesopotamischen Heiler, ist nik/qiptu vielleicht aus eben diesem Grund der (wohl für eine Pflanze stehende) Begriff „Hundekot“ an die Seite gestellt..$^{55}$

Auch wenn - wie es Erica Reiner annahm ${ }^{56}$ - der akkadische Pflanzenname nik/qiptu ein Lehnwort aus der sumerischen Bezeichnung ligidba oder aber - wie Thompson meinte ${ }^{57}$ - semitischen Ursprungs und zu dem syrischen Verb $n^{e} q a b h$,durchbohren" zu stellen ist, glaubten die mesopotamischen Heiler selbst, in dem Klang der Bezeichnung ihres Arzneimittels dessen Wirkung zu erkennen. Da sie ausgerechnet zur Heilung einer nikipti Sîn genannten Kinderkrankheit, deren Name wörtlich „Stoß des (Mondgottes) Sîn“ bedeutet, die nik/qiptu geheißene Arznei „Ninurta-Duft“ einzusetzen empfahlen, ${ }^{58}$ dürften sie auch eine etymologische Verbindung zwischen dem akkadischen Wort für Stoß (nikiptu) und der Bezeichnung des Heilmittels gesehen haben. Die im Alten Orient der Arznei offensichtlich beigemessene Kraft, so wie der Gott

ebenfalls vor Schadenzauber schützen (siehe z. B. Abusch/Schwemer 2011: 188, 43'-45'; Schuster 2008, 129 Kette 109). Gegen Ohrenbrausen, das als ein vom Totengeist verursachtes Symptom galt, wurde es gemeinsam mit anderen pflanzlichen Heilmitteln zur Räucherung verwendet (Thompson 1949, 365; Scurlock 2006, 271-273 Nr. 60; ähnlich ebd., 350 Nr. 117; 390 f. Nr. 137 a und b; 393 Nr. 138; 563 Nr. 248; 569 Nr. 253; 588 Nr. 272; 601 Nr. 285; 602 Nr. 286; siehe auch Parpola 1983, 250-253). Auch in Verbänden (um Kopfschmerzen [sAĜ.KI.DAB. DA] zu lindern: Heeßel 2006, 24: 10-11; siehe auch Scurlock 2006, 282 Nr. 69), getränkten Umschlägen (lippu für das Ohr: Scurlock 2006, 423f. Nr. 163 a und b) und Tampons (für das Ohr: Scurlock 2006, 411 Nr. 153) kam es zum Einsatz. Um gegen Schadenzauber Wirkung zu entfalten, wurde es auch innerlich verabreicht und Tränken beigegeben (Abusch/Schwemer 2011, 235: 132'"'-140"') oder zum Essen verordnet (ebd., 412: 13'-19'). Darüber hinaus setzte man es Waschund Weihwassern (Maul 1994, 42 und 462: 1'-4'; Dick 1999, 102: 35) und auch den im Tempel verwendeten Räucherwerkmischungen zu (Jursa 2009, 148).

53 So Thompson $(1949,366)$.

54 Siehe zuletzt Kinnier Wilson (2005).

55 Siehe Uruanna III 39: Ú ni-kip-ti Aš zê kalbi lišān kalbi eșemti kalbi (zitiert nach CAD N/2 222 s. v. nikiptu A).

56 Reiner 1995, 35 Anm. 144. Thompson hingegen sah sowohl in der sumerischen als auch in der akkadischen Namensform ,varied versions of a foreign word“ (Thompson 1949, 367 Anm. 1).

57 Thompson 1949, 367.

58 Labat 1951, 222: 42; Stol 1998, 344 sowie ders. 2000, 211 zu 42f.
Uta'ulu/Ninurta im Kampf gegen die Monster „Stöße“ auszuteilen, wird man vor allem in ihrem starken Geruch zu suchen haben. Wohl nicht zuletzt, um eine Analogie zu der Vorstellung von einem aggressiven männlichen und einem sorgenden weiblichen Schutzgeist herzustellen, wurden in der Salbe des Heilers beide Arten des „NinurtaDuftes" verwendet. Während sich in der männlichen die Kraft des Kämpfergottes Bahn bricht, steht die weibliche (möglicherweise weniger intensiv riechende) Form für die Macht der dem Heldengott an die Seite gestellten Göttin Ningirima. Das dem Heiler auf Schritt und Tritt (siehe Z. 8) Schutz gewährende Götterpaar Uta'ulu und Ningirima umgibt somit seinen Schützling tatsächlich von Kopf bis Fuß am ganzen Leib und strahlt seine Kraft in der Gestalt des Geruchs - ganz so wie es in dem zu rezitierenden sumerischen Text gesagt ist (Z. 5-11) - in alle Richtungen aus.

An seinem „Ninurta-Duft“ dürfte man einen mesopotamischen Heiler bei der Arbeit bereits aus einiger Entfernung deutlich wahrgenommen haben. Unwillkürlich denkt man an den starken Geruch des Kampfers, der aus Bäumen (cinnamomum camphora) gewonnen wird und antiseptische Wirkung besitzt, ${ }^{59}$ aber auch an die Pestarztmasken der frühen Neuzeit, in deren Schnabel man einen mit duftenden Essenzen getränkten Schwamm plazierte, um dem ,Miasma‘ etwas Positives entgegenzusetzen. Der Einsatz des stark riechenden nik/qiptu ist dementsprechend als Versuch der Heiler zu werten, der Gefahr der üblen Luft von Unheil und Krankheit entgegenzuwirken.

Einige Verse aus dem sog. Weltschöpfungsepos Enūma eliš zeigen, dass die Heiler auch mit derartigem Vorgehen gegen die giftigen Ausdünstungen von Krankheit und Unheil glaubten, es Asalluhi, dem Gott der Beschwörungskunst, gleichzutun. Denn in der vierten Tafel von Enūma eliš ist geschildert, dass auch der mit Asalluhi gleichgesetzte Marduk im vorweltlichen Urkampf mit einem pflanzlichen Mittel gefeit seiner großen Gegenspielerin Tiamat entgegentrat:

Auf Tiamat, die in voller Wut war, richtete er da seinen Blick. Auf seinen Lippen hielt er dabei das beschwörende Wort, das Kraut, um Gift auszulöschen, fest umfassend mit seiner Hand..$^{60}$

59 Siehe van Wyk [u.a.] (2004, 104).

60 Enūma eliš IV 60-62: ašriš Tiāmat ša uggugat pānuššu iškun / ina šaptišu tâ ukalla / šammi imta bullî tameh rittuššu. Nach dem hier beschriebenen Vorbild des Marduk ließen sich im 7. Jh. neuassyrische Könige und hochgestellte Mitglieder der Königsfamilie mit einem solchen „Kraut“ in der Hand darstellen (siehe Lambert 2013, 88). 
Hier ist zweifellos der mythische Ur-Exorzismus geschildert, der sich in der täglichen Arbeit der Heiler wiederholte.

In einer Beschreibung des bit rimki („Badehaus“) genannten umfänglichen Reinigungsrituals, das „Beschwörer“ zum Schutz des Königs durchzuführen pflegten, ${ }^{61}$ sind ausnahmsweise einmal jene den Heilern selbstverständliche und daher in ihrem Schrifttum nur selten erwähnte Handlungen beschrieben, denen sie sich zum eigenen Schutz regelmäßig zu unterziehen hatten, wenn sie gegen Übel, Unheil und Krankheit vorgehen wollten. Fast wörtlich entsprechen diese Handlungsanweisungen der aus VAT 8803, Rs. $21 \mathrm{f}$. bekannte Passage:

Der „Beschwörer“ (mašmaššu) zerstößt männliches und weibliches nik/qiptu (und) vermischt das mit Honig und Butterschmalz. Er salbt sich damit ein. ${ }^{62}$

In der Beschreibung des bìt rimki-Rituals ist der „Beschwörer“ im folgenden auch angehalten, rote Gewänder (nahlaptu sāmtu und șubātu sāmu) anzulegen, um - auf diese Weise gefeit - gegen das Unheil angehen zu können. Hieran sehen wir zum einen, dass die Salbe bereits vor dem Anlegen der Kleider aufgetragen wurde. Zum anderen zeigt diese Passage, dass der Heiler, wenn er gegen Krankheit und Übel vorging, eine spezielle Kleidung zu tragen hatte, die - wie wir aus der Überlieferung der „Beschwörer" wissen, mit ihrer roten Farbe die üblen Geistwesen erschrecken und in die Flucht schlagen sollte. ${ }^{63}$ Darstellungen der „Beschwörer“ bei ihrer Arbeit, lassen erken- nen, dass ihre Kleidung - oft in der Gestalt einer mannsgroßen Fischhaut mit dem Fischkopf als Kappe - sie fast vollständig einhüllte und so wohl tatsächlich ähnlichen Schutz vor Ansteckung bieten sollte, wie die Schutzkleidung, die heute auf Infektionsstationen Verwendung findet. Die in der Beschreibung des bit mēseri genannten Verfahrens anzutreffende Bemerkung, der Schmiedegott Nin-á-gal gehe immer wieder dem „Beschwörer“ voran, ${ }^{64}$ kann man sogar als Hinweis darauf verstehen, dass bisweilen auch ein metallenes Pektorale o.ä. zu der schützenden Ausstattung eines Heilers gehörte.

Welch große Bedeutung die Heiler ihren Vorsichtsmaßnahmen beilegten, lässt sich an einer Warnung ermessen, die sich in der Einleitung eines unter den „Beschwörern“ im ersten Jahrtausend weit verbreiteten ,Handbuchs' findet. Das keilschriftliche Kompendium in Omenform, in dem zur Bestimmung eines Leidens und zur Ermittlung der jeweils sachgerechten Therapie zahllosen Symptombeschreibungen Angaben über die Art der zugehörigen Krankheit und deren Heilungschancen gegenübergestellt sind, beginnt nämlich mit der Mahnung:

Wenn du dich dem Kranken näherst, darfst du so lange nicht an ihn herantreten, um ihn zu heilen, bis du die ,Beschwörung‘ über dich ,geworfen' hast. ${ }^{65}$

Es besteht kein Zweifel daran, dass mit dieser ,Beschwörung' der hier besprochene und im folgenden in Bearbeitung vorgelegte Text gemeint ist.

\section{Der Text}
$\mathrm{A} \quad=$ VAT 8803
Z. 1-33; Kolophon
Kopie: E. Ebeling, KAR I, 56 f. Text Nr. 31; Bearbeitung: E. Ebeling, ZDMG 69 (1915) 89-103; Nachtrag: B. Meißner, ebd., 412-414. Photos: oben S. $178 \mathrm{f}$.
$\mathrm{B}=\mathrm{K} 9836+\mathrm{K} 10338+\mathrm{K} 20638$ Photo: CDLI P398343; Bearbeitung: -
$\mathrm{C}=\mathrm{K} 10565$, Vs Photo: CDLI P398758; Bearbeitung: -

61 Siehe Laessøe (1955) und zur Einführung Farber (1987, 245-255). 62 BBR, Text Nr. 26: i, 23-25: mašmaššu(MAš.MAš) nik/qiptu(šıM. $\left.{ }_{\text {dMAŠ }}\right) z i k a r(\mathrm{NITA}) u$ sinniš(MUNUS) / i-su-ak-ma itti(KI) dišpi(LÀL) himēti(İ.NUn.NA) iballal(HE.HE) / ip-pa-šáa-áš. Vgl. auch die Parallelstelle ebd., ii 5-7: lúMAŠ.MAŠ / ŠIM. ${ }^{\mathrm{d} M A S ̌ ~ N I T A ~} u$ MUNUS $i$-su-ak-ma / KI LÀL İ.ĜIŠ İ.NUN.NA HE.HE ŠÉŠ.

63 BAM 8, 298: $35 \mathrm{f}$.
64 ina 'pa-an' mašmašši([M]Aš.MAš) ittanallaka(DU.DU) d $N[$ in-á-gal]: Meier 1941-44, 146: 117 = VAT 13666 + VAT 13680, ii 21' (kollationiert). 65 Labat 1951, 16 f.: 85 und 18f.: 1 und Anm. 27; Heeßel 2000, 71 mit Anm. 12. 
1 A Vs. 1 [ÉN $\hat{g}$ ]á-e lúkị́̂-gi ${ }_{4}$-a dị̂̂ir gal-gal-e-ne-me-en $[m a] r$ šip-ri ša il(ān)ì(DIĜIR.MEš) rabûti(GAL.MEŠ) ana-ku

2 A Vs. 3 [d+en-k]i dasal-lú-ḩi gal-bi á mu-un-da-an-á $\hat{g}$

4 [d] 'é ${ }^{1}-a$ ù ${ }^{\mathrm{d}}$ marduk(AMAR.UTU) rabîš(GAL-iš) ú-ma-'i-i-ru-in-ni

3 A Vs. $5{ }^{\top} \mathrm{tu}_{6}{ }^{\top}-\mathrm{t} \mathrm{u}_{6}$-šè al-du ki silim-ma šu-ĝ $\mathrm{u}_{10}$ ús-en

B Vs. 1' [ ] 'ki silim'-ma š[u-

C Vs. $1^{\prime} \quad\left[\mathrm{tu}_{6}-\mathrm{tu}\right]_{6}$-šè al-du- ${ }^{\top} \mathrm{unn}^{\top} \mathrm{k}[\mathrm{i}$

A Vs. 6 i-na šip-ti al-lak a-šár šu-ul-me qa-ti um-mad

B Vs. 2' [ ]a-šar šul-me [

4 A Vs. 7 tu $u_{6}$ kù-ga-bi ka-ĝ $u_{10}$-ta mu-un-da-ĝál

B Vs. 3' [t $\left.u_{6} k \grave{u}-g a-b i\right] k a-\hat{g} u_{10}-t a$ [

C Vs. 2' $\mathrm{t}\left[\mathrm{u}_{6}\right]$ kù-ga-bi ka- $\hat{\mathrm{g}}\left[\mathrm{u}_{10}-\mathrm{ta}\right.$

A Vs. 8 ši-pat-su-nu elletu(kÙ-tu) na-šá-ku ina pi-ia

B Vs. 4' [ši-pat-su-n]u elletu(kÙ-tum) na-šá-ku [ ]

5 A Vs. 9 duta-u ${ }_{18}-l u$ ur-sâg diĝir-re-e-ne-ke 4

B Vs. 5' [ ] ur-sâg diĝir-[re-e-ne-ke $\left.{ }_{4}\right]$

C Vs. 3' ${ }^{\top \mathrm{d}} \mathrm{u} \mathrm{ta}^{\top}-\mathrm{u}_{18}-\mathrm{lu}$ ur-s[a $\hat{\mathrm{g}}$

A Vs. 10 dninnu(50)-urta qar-rad il(ān)ì(DIĜIR.MEš)

B Vs. 6' [ ] qar-rad [

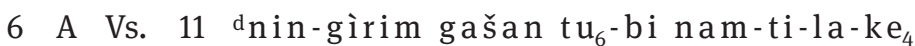

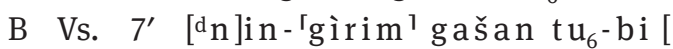

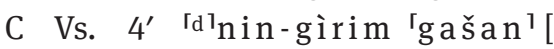

A Vs. 12 dMIN be-el-tum šá tu-ú-ša ba-la-țu

B Vs. $8^{\prime} \quad{ }^{\mathrm{d}} \mathrm{MIN}$ be-el-tum šá tu-šá [

7 A Vs. 13 igi egir zi-da á gùb-bu-da

B Vs. 9' [ ] egir zi-da 'á gùb-bu'-[da]

C Vs. 5' igi egir zi-da 'á? [

A Vs. 14 pa-na ar-ka im-na u šu-me-la

B Vs. 10' pa-na ar-ka [

8 A Vs. 15 lú huul ba-an-šar(„EZEN“)-re-eš ki-ús-sa-ĝ $u_{10}$ ùri aka-eš

B Vs. 11' [lú] huul ba-an-šar-re-eš [

C Vs. 6' lú 'hŭul ba-an-šar-re-eš']

A Vs. 16 lem-na i-țàr-ra-dui-na-șa-ru kib-si

B Vs. 12' lem-nu i-țar-ra-d[u

9 A Vs. 17 igi ĝal $1_{5}$-lá ki kúr-šè ba-an-ĝar-re-eš

B Vs. 13' [ig]i 'îgal ${ }_{5}{ }^{\top}-1$ á ki kúr-š [è

(abgebrochen)

C Vs. 7' igi ĝal $1_{5}$-lá ki kúr-š[è

A Vs. 18 pa-an gal-le-e ana âšsi šá-nim-ma i-šak-ka-nu 
10 A Vs. 19 ki nam-ti-la-ke 4 á-ĝ $u_{10}$-ta ba-an-gub-bu-uš

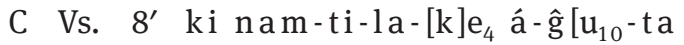

A Vs. 20 i-na qaq-qar ba-lá-ți i-da-a-a iz-za-az-zu

C Vs. 9' i-na qaq-qar ba-lá-ți ${ }^{[} i^{1}-[d a-a-a$ ]

11 A Vs. 21 an-ta ki-ta ki silim-ma ba-ra-ab-ĝá-ĝá-aš

C Vs. 10' an-ta ki- 'ta ki silim ${ }^{1}-[\mathrm{ma}$

A Vs. 22 e-liš u šap-liš $a$-šár šu-ul-mi(über-me) iš-ku-nu

C Vs. $11^{\prime} e$-liš $u[$

12 A Vs. 23 tu $u_{6}$ kù-ga-bi ba-ra-ab-sum-mu-uš

C Vs. $12^{\prime}{ }^{\mathrm{T}} \mathrm{tu}_{6}{ }^{\top} \mathrm{k}[\mathrm{u}$ -

(abgebrochen)

A Vs. 24 ši-pat-su-nu elletu(кù-tum) id-di-nu-nim-ma

13 A Vs. 25 inim mu-un-da-ab-dug-ga-ašan-da-ab-aka-a-me-en

26 a-mat i-qab-bu-u-ni ep-pu-uš

14 A Vs. 27 nam-mah e-ne pa è aka-me-en

28 nàr-ba-šu-nu ú-šá-apa?(Áв)-ma

15 A Vs. 29 udug $\operatorname{sig}_{5}$-ga dlamma sig ${ }_{5}$ ga há-en-da-su $u_{8}$-su $u_{8}$-ge-eš

30 še-ed dum-qí la-mas-si dum-qí lit-tal-la-ku it-ti-ia

16 A Vs. 31f. a-lá máškim dlugal-ùr-ra(-)an-ta(-)šub-ba〈an-〉ta-šub-ba / saĝ-hul-hna-za

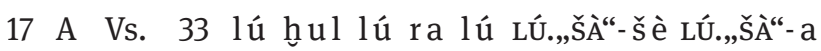

34 lem-nu ka-mu-ú ḩab-bi-lu šag-gi-šú

18 A Vs. 35 dnam-tar lú níĝhul nu dùg-ga ba-an-gub-ba

36 dMIN šá ana le-mut-ti u la ța-ab-ti iz-za-az-zu

(Rand)

19 A Rs. 1 ní ĝ-nam su lú-ka ĝál-la ù ĝ kalam-ma ba-ba

2 mim-ma šum-šú šá ina zu-mur ni-ši šak-nu-ma ni-iš ma-a-ti ú-na-âš-šá-ru

20 A Rs. 3 sil $-1 a ́$ sil $-1 a ́$ bad-rá bad-rá gaba-zu tu-lu-ub

4 pu-țur dup(,„UM“')-piri-si re-e-qí i-rat-ka né-’i

B Rs. $1^{\prime}$ [ né]-[' ${ }^{\prime} i^{1}-[i]$

21 A Rs. 5 šu-zu tùm-ma-ab gì̀ri-zu zi-ga-ab

B Rs. 2' [ z]i-ga-[ab]

A Rs. 6 ta-bal qātt(šu)-ka ú-su-uh še-ep-ka

B Rs. 3' [ š]e-ep-[]

22 A Rs. 7 ki kúr-šè ĝen-na a-ga-zu-šè gi $\dot{i}_{4}$-bí-í[b]

B Rs. 4' [ ] gi-bí-í[b]

A Rs. 8 a-na âš-ri šá-nim-ma at-lak ana âr-ki-ka tu- ${ }^{-} u{ }^{1}$

B Rs. 5' [ -k]a tu-ur 
23 A Rs. 9 sil $1_{7}$-lá igi- $\hat{\mathrm{g}} \mathrm{u}_{10}$-ta $\mathrm{sil}_{7}$-lá egir-ĝ $\mathrm{u}_{10}$-ta

B Rs. 6' $[$ eg $]$ ir- $\hat{\mathrm{g}} \mathrm{u}_{10}-\mathrm{ta}$

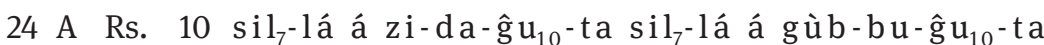

$\mathrm{B}$ Rs. $7^{\prime}[\quad \mathrm{gùb}-\mathrm{b}] \mathrm{u}-\hat{\mathrm{g}} \mathrm{u}_{10}-\mathrm{t}[\mathrm{a}]$

25 A Rs. 11 an-ta ki-ta nam-mu-un-DU-nitgin-e

B Rs. 8' [ ]- 'niĝin-e ${ }^{1}$

A Rs. 12 e-liš u šap-liš e ta-as-sahn-ra

B Rs. 9' [ ] ' e ta ${ }^{1}-[a s-s a h]^{-}{ }^{\top} r{ }^{\top}$

26 A Rs. 13 ĝá-e lú-kîĝ-gi $i_{4}-a^{d}+e n-k i d a s a l-l u ́-h i-m e-e n$

B Rs. $10^{\prime}[$ ] d +en-ki d [

A Rs. 14 mar šip-ri šá dé- $a u$ d marduk(AMAR.UTU) $a n a-k u$

B Rs. 11' [ ] $\quad$ šá dée- $a^{\top} u^{\mathrm{d}}{ }^{\top}[$

27 A Rs. $15 \mathrm{mu}$ pàd-da-bi-šè záh̆-ab

B Rs. 12' [ ]záh̆-[ ]

A Rs. 16 ana zi-kir šu-me-šú-nu na-âr-qí

B Rs. 13' [ -n]u nar-[ ]

28 A Rs. 17 zi duta- $u_{18}-1 \mathrm{u}$ ní ù-bí-ta nam-mu-un-da-te-ĝ $e_{26}-\mathrm{e}-\mathrm{d} e ̀$

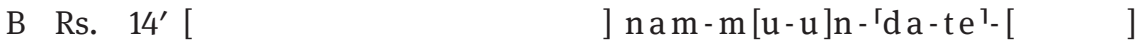

A Rs. 18 ni-iš ${ }^{\mathrm{d} M I N}$ pi-làh-ma la te-țe-eh-ḩa-a (Strich)

B Rs. $15^{\prime}[$-m]a la $[\quad]$

(Strich)

29 A Rs. 19 ka-ini m-ma gallû(GAL -LÁ) rābiṣu(MÁŠKIM) dlugal-ùr-ra saĝ-ḩul-ḩa-za alû(A.LÁ) lemnu(HUL)

B Rs. $16^{\prime}[$

] 'MÁŠKIM d'[

30 A Rs. 20 antašubbû(AN.TA.šUB.BA) mim-ma šum-šú ana mašmašši(MAš.MAš) lā(NU) țehê(TE-e) (Strich)

B Rs. $17^{\prime}$ [AN.TA.šUB].BA u mim- ${ }^{\top} m a$ šum-šu ${ }^{1}[$

(Strich)

31 A Rs. 21 d ù-dù-bi nik/qiptu(ك̌IM.dMAš) zikar(NITA) u sinniš(MUNUS) tasâk(SÚD) ina dišpi(LÀL) $u$ himēti(ì.NUN.NA) taballal(HE.HुE)

B Rs. $18^{\prime}\left[\mathrm{d} \grave{\mathrm{u}}-\mathrm{d} \grave{\mathrm{u}}-\mathrm{bi} \quad\right.$ ŠIM. $\left.{ }^{\mathrm{d}} \mathrm{N}\right]$ IN.URTA NITA $u \quad$ MUNUS S[ÚD

32 A Rs. 22 e-nu-ma ana lúmarși(GIG) te-țè-eh-huu-ú ra-man-ka ištēniš(DIŠ-niš) tapaššaš( ̌̌Éš)-ma

B Rs. 19' [e-nu-ma ana lúGI ${ }^{\prime} \mathrm{G} \quad$ TE-ú $\quad r[a-m a n-k a$

(abgebrochen)

33 A Rs. 23 ana lúmarși(GIG) tețehhe(TE-ḩe) mim-ma lem-nu lā(NU) ițehhēe(TE)-ka (Strich) 


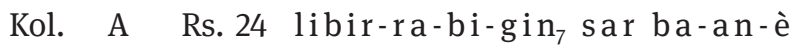

$25 q \bar{a} t(\check{S} \mathrm{U})$ I.dna-bi-um-be-sún

26 mār(DUMU) I.dba-ba $a_{6}$-šuma(MU)-ib-ni maš-maš bit(té) kiš-šú-ti

27 tābil(TÙM) țuppi(IM) šuāti(BI) d+nabû(AG) $u$ dnissaba bēlū(EN.MEš) bīt(É) mu-um-me

28 țu-mu-mì-iš $i$-šim-mu-šu

(Rand)

\section{Übersetzung}

1 [Beschwörung]: Ich bin es, der Bote der großen Götter! -

2 Enki unterwies den? Asalluhi mit großer Sorgfalt. akk.: Ea und Marduk unterwiesen mich mit großer Sorgfalt.

3 Um der beschwörenden Worte willen gehe ich einher, ich lege (dabei) meine Hand auf eine Stelle des Heils. akk.: Mit der Beschwörung versehen gehe ich einher, ich lege (dabei) meine Hand auf eine Stelle des Heils.

4 Jenes heilige beschwörende Wort liegt mir im Munde. akk.: Ihre heilige Beschwörung trage ich im Munde.

5 Uta’ulu, der Vorkämpfer der Götter, akk.: Ninurta, der Vorkämpfer der Götter,

6 Ningirima, die Herrin, deren beschwörendes Wort das Leben selbst ist,

7 vertrieben vorne, hinten, rechts und an der linken Seite akk.: vertreiben vorne, hinten, rechts und links

8 den Bösewicht und wachten darüber, wohin ich trat. akk.: den Bösen und behüten meinen Wandel.

9 Den Blick des galla-Dämonen richteten sie aus auf einen fremden Ort. akk.: Die Aufmerksamkeit des gallû-Dämonen richten sie auf einen anderen Ort.

10 Auf einem Boden (getränkt) mit Leben stehen sie mir zur Seite.

11 Oben und unten bereiteten sie mir einen Raum des Heils.

12 Jenes heilige beschwörende Wort übergaben sie mir. akk.: Ihre heilige Beschwörung übergaben sie mir, damit

13 Sprachen sie in einer Angelegenheit ein Wort, bin ich es, der es ausführt. akk.: ich das Wort, das sie zu mir sprechen, ausführen kann.

14 Ich bin es, der in ihrer Erhabenheit sie strahlend sichtbar werden lässt. akk.: Ihre großen Taten lasse ich strahlend sichtbar werden, auf dass

15 Ein guter Geist und eine gute Schutzpatronin mögen mich stets begleiten! akk.: ein guter Geist und eine gute Schutzpatronin mich stets begleiten!

16 Ala-Dämon, „Lauerer“, „,on oben herabgefallener Herr des Daches“, „Der von oben Herabgefallene“, „Der das Böse unterstützt“,

17 Bösewicht, Fessler, Mörder, Übeltäter, akk.: Böser, Fessler, Übeltäter, Mörder,

18 Todesbote, der wegen böser, wegen unguter Dinge schon bereit steht,

19 oder irgendetwas sonst, das sich im Leib eines Menschen befindet und den Leuten des Landes Schaden zufügt akk.: oder irgendetwas sonst, das in den Leib der Leute gelegt ist und dann den Leuten des Landes Schaden zufügt -

20 weiche zurück, weiche zurück! Halte dich fern, halte dich fern! Deine Brust dreh nach hinten! akk.: löse dich, weiche zurück, entferne dich, verschwinde! Deine Brust dreh' nach hinten!

21 Deine Hand zieh zurück! Deinen Fuß hebe fort!

22 Mach dich davon an einen fremden Ort! Kehre zurück dorthin, woher du kamst! akk.: Mach' dich davon an einen anderen Ort! Kehre zurück dorthin, woher du kamst!

23 Weiche vor mir zurück, weiche hinter mir zurück!

24 Weiche zurück von meiner rechten Seite! Weiche zurück von meiner linken Seite!

25 Weder von oben, noch von unten sollst du mich umschwirren! 
26 Ich bin es, der Bote von Enki (und) Asalluhi!

akk.: Ich bin es, der Bote von Ea und Marduk!

27 Verkriech dich, wenn diese Namen ausgesprochen werden!

akk.: Verkriech dich, wenn ihr Name ausgesprochen wird!

28 Das Leben des Uta'ulu möge dich so in Angst versetzen, dass du mir nie mehr nahe kommen wirst!

29 f. Wortlaut der Beschwörung, (zu rezitieren,) damit der gallû-Dämon, der „Lauerer“, der „Herr des Daches“, „Der das Böse unterstützt“, der böse alû-Dämon, „Der von oben Herabgefallene“ oder irgendetwas sonst sich dem Beschwörer nicht nähern kann.

31-33 Die zugehörigen Handlungen: Du zerstößt männliches und weibliches nik/qiptu (und) vermischst das mit Honig und Butterschmalz. Wenn du dich dem Kranken näherst, salbe dich selbst damit überall ein, und dann kannst du dem Kranken nahe kommen. Jegliches Übel kann sich dir nicht mehr nähern.

Kol. A Wie die zugehörige Vorlage geschrieben und kollationiert.

Hand des Nabû-bēssun, des Sohnes des Baba-šuma-ibni, des „Beschwörers des Hauses der Allmacht“. Wer diese Tafel fortträgt, dem mögen Nabû und Nissaba, die Herren des bït mumme, ein Schicksal das dem eines Tauben gleicht, bestimmen.

\section{Kommentar}

2 Die beiden Fassungen der Zeile weichen erheblich voneinander ab. Die sumerische Version des Textes spiegelt wohl (trotz der fehlenden Kennzeichnung des Ergativs) die in der sog. Marduk-Ea-Formel beschriebene Situation (siehe Falkenstein 1931, 53-58): Enki, der Weisheitsgott, instruiert seinen göttlichen Sohn Asalluhi, damit dieser Wissen und Kunst seines Vaters zu den Menschen bringe. Während die sumerische Zeile die göttliche Perspektive der Historiola der Beschwörungen vom ,MardukEa-Typ“ vermittelt, betont die akkadische Übersetzung die menschliche. Der Instruierte ist hier nicht der Gottessohn, sondern der Heiler und Beschwörer, der āšipu bzw. mašmaššu, der in seinen Therapien de facto als Enki-Sohn Asalluhi agiert (siehe dazu Maul 1994, 41).

5-8 Dem Wunsch, stets von allen Seiten von Göttern oder engelhaften Wesen geschützt und so vor allem Bösen gefeit zu sein, ist in der Literatur der „Beschwörer“ (āšipūtu) immer wieder Ausdruck verliehen (siehe z.B. Finkel 2000, 199f. Text 43; BAM 8, 104-106: 66-71; 111-113: 107-112; 126f.: 170-177 und 128: 180 f. sowie Mayer 1976, 244-249).

14 In babylonischen Gebeten ist die Verbindung von narbû mit $(w) \operatorname{apu}(m)$ Š-Stamm so geläufig (siehe Mayer 1976, 325), dass diese auch hier vorliegen muss - trotz der eigentümlichen Schreibung ú-šá-ÁB-ma, die E. Ebeling noch ú-šá-lid-ma gelesen hatte (Ebeling 1915, 90). ${ }^{66} \mathrm{~W}$. von

66 B. Meißner hatte bereits 1915 Ebelings Lesung korrigiert (Meißner 1915, 412).
Soden (siehe AHw. 1460a zu dieser Textstelle: narbâ-šunu ú-šá-pa!) war wohl davon ausgegangen, dass entweder in E. Ebelings Autographie in KAR Nr. 31 oder aber in der Textabschrift des Nabû-bēssun(u) statt ú-šá-pa fehlerhaft ú-šá-ÁB geschrieben wurde. Nimmt man jedoch, was nahe liegt, an, dass das Zeichen Áв hier / apa/gelesen wurde, ist eine entsprechende Emendation unnötig (bei von Soden/ Röllig 31976, 47 ist der Lautwert apa für das Zeichen ÁB jedoch ebensowenig gebucht wie bei Borger 22010, 396 s. v. 672 ÁB).

$15 \mathrm{Zu}$ den Schutzgenien šèdu und lamassu siehe von Soden (1964), Vorländer (1975, 25 f. und 47 f.), Mayer (1976, 244-249), Groneberg (2006) sowie die Zeilen 15 und 41-48 der ersten Tafel des Preisliedes auf Marduk ludlul bēl nēmeqi (siehe Annus/Lenzi 2010, 15-16 und Oshima 2014, 78 und 80). Der Wunsch, schützende Geister auf Dauer zur Linken und zur Rechten zu haben, manifestiert sich auch in einem Paar goldener Ohrringe aus altbabylonischer Zeit (Marzahn 2008, 316 Abb. 235 und 317 Nr. 323), das die Schutzgöttin Lamma zeigt.

16 In dieser Zeile sind fünf Unheil stiftende Geistwesen genannt, die von einem Menschen Besitz ergreifen und damit bedrohliche Krankheiten bewirken können. Vom a-lá-Dämon (akkadisch: alû) heißt es u.a., er „packe den Mund“ eines Menschen, so dass dieser nicht mehr sprechen kann. Die uns zugänglichen Symptombeschreibungen hält M. Stol für die Beschreibung einer „form of stroke“ (Stol 1993, 41). Es bleibt unklar, ob auch das Wirken des „Lauerers“ (máškim = rābișu) mit einem konkreten Krankheitsbild verbunden war. dlug a l-ùr - r a jedoch galt als ein dämonenhaftes göttli- 
ches Wesen, dessen Wirken an einem der Epilepsie zuzuordnenden Feld von Symptomen zu erkennen ist, die sich allesamt an den nicht mehr unter Kontrolle gehaltenen Augen eines Kranken zeigen (Stol 1993, 16-19). In akkadischem Kontext konnte der sumerische Name, der „Herr des Daches“ bedeutet, sowohl dlugal-ùr-ra als auch bēl ūri $(m)$ gelesen werden (siehe Borger 22010, 307). In Z. 16 ist dlugal-ùr-ra(-)an-ta(-)šub-ba als eine Einheit $\mathrm{zu}$ verstehen, deren akkadische Entsprechung miqit bēl ūri lautete (Stol 1993, 17 mit weiterführenden Angaben). miqtu, „Fall“, „etwas, das herabgefallen ist“, bezeichnet häufig eine Krankheit, die nach M. Stol als ein plötzliches Gepacktwerden wahrgenommen wurde (Stol 1993, 11) und in vielen Fällen wohl mit der Epilepsie gleichzusetzen ist (Stol 1993, 9). Das Krankheitsbild der Epilepsie konnte auch mit dem in unserer Zeile folgenden gelehrten sumerischen Namen a n-t a (-)šu b - b a (akkadisch: miqit šamê, „vom Himmel Gefallenes“) bezeichnet werden (Stol 1993, 7-9). Auch wenn wir s a ĝ- h u l- h a - z a (akkadisch neben saghulhazû, saghulhazakku auch: mukīl rēš lemutti(m), „Der das Böse unterstützt“) bislang mit keinen spezifischen Symptomen verbinden können, scheint es, dass die in Z. 16 erscheinende Folge von ,Dämonen' für den Verlauf einer sich verschlimmernden Erkrankung steht, die im weitesten Sinne als Kontrollverlust über die eigene Person beschrieben werden kann, aber nicht zwangsläufig unserer Definition von Epilepsie entsprechen muss.

$17 \mathrm{Zu}$ diesem Beleg für LÚ.,,̌̌̀̃“-š è anstelle von LÚ×GÁNAtenû siehe Attinger $(1993,595)$.

18 In seiner Autographie des Textes VAT 8803 (KAR I, S. 56, Z. 35) hat E. Ebeling das Zeichen Ní̂́ in ní $\hat{\mathrm{g}}$ - hu ul versehentlich ausgelassen.

23f. Die Zeilen 23 und 24 versah Nabû-bēssun(u), der Schreiber des Textvertreters A, nicht eigens mit einer akkadischen Interlinearübersetzung, da sich diese aus den weitgehend parallelen, jeweils ins Akkadische übertragenen Zeilen 7 und 20 erschließen lässt.

28 Uta'ulu wurde schon früh mit dem Enlil-Sohn Ninurta gleichgesetzt, der zahlreichen Mythen zufolge als „Rächer seines Vaters“ durch kriegerische Heldentaten die Welt vor Unheil und Untergang bewahrte. Der Gott Uta'ulu = Ninurta ist hier nicht zufällig genannt. Denn der Ritualanweisung Z. 31-33 zufolge ist es die „Ninurta-Duft“ (ŠIM. ${ }^{\mathrm{d} N I N . U R T A}$ oder ŠIM. ${ }^{\mathrm{d}}$ MAŠ) genannte Droge nikiptu (bzw. niqiptu), durch die die in Z. 28 beschworene Lebenskraft des Gottes deutlich wahrnehmbar wird. In Z. 28 steht ní ta für ní te = palāhu .

32 In seiner Autographie des Textes VAT 8803 (KAR I, S. 57) hat E. Ebeling die Zeichenfolge DIŠ-niš = ištēniš versehentlich ausgelassen. Daher fehlt in den Wörterbüchern der hier vorliegende Beleg für ramānka ištēniš tapaššaš. Die Wendung ištēniš pašāšu ist sonst nur äußerst dürftig belegt (Oppenheim 1956, 339: x+16; Scurlock 2006, 605 f., A $77 \mathrm{f}$.$) .$

Kol. Eine Bearbeitung des Kolophons findet sich in Hunger 1968, 67 Nr. 192. Es ist keineswegs sicher, dass bei der Lektüre der Tafel der Verweis libir-ra-bi-g in $\mathrm{s}_{7} \mathrm{sar}$ ba-an-è (A Rs. 24) ins Akkadische übertragen wurde (kïma labīiřšu šațir(ma) bari).

Der Titel mašmaš bìt kiššūti, „Beschwörer des Hauses der Allmacht" ist nur an dieser Stelle bezeugt (siehe Maul 2010, 203). „Haus der Allmacht“ ist, so wie É-šár-ra („Haus des Alls“), ein Prunkname des Assur-Tempels. Nach der im Fach ,Götteradressbuch ' genannten Liste galt bit kiššüti als akkadische Übersetzung des sumerischen Tempelnamens É-šár-ra (siehe Menzel 1981, T 156: 144; George 1992, 176: 144). Bāba-šuma-ibni ist sonst der Titel mašmaš bit Aššur, „Beschwörer des Assur-Tempels“ zugewiesen. Es ist daher nicht unwahrscheinlich, dass beide Titel unterschiedslos die gleiche Stellung bezeichneten.

Nabû und Nissaba, Gott und Göttin der Gelehrsamkeit, gelten hier als die Herren des bit mumme, jenes heiligen Ortes, an dem im Assur-Tempel die Götter(bilder) entstanden. Der Wunsch, dass die Weisheitsgötter den Tontafeldieb „taub“ werden lassen, ist für den Gelehrten ein schlimmer Fluch, denn taub sein meint hier, mangels der Fähigkeit zu ,hören', dauerhaft auf jegliche Inspiration der Weisheit verzichten zu müssen und so den Zugang zu Einsichten aller Art zu verlieren.

\section{Anhang: K 10565 (Photo: CDLI P398758), Rs.}

Rs. $1^{\prime}$ f. (Spuren)

3' [lú] 'hull igi [hul ka huul eme hul]

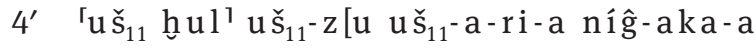
níĝ-huul-dím-ma ]

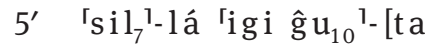

6' (Spuren)

7' z [i an]-na ḩé-p[àd zi ki-a hué-pàd]

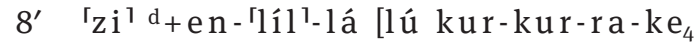
hé - pàd ]

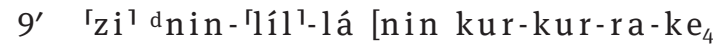
hyé-pàd]

$10^{\prime} \mathrm{zi}^{\mathrm{d}}+\mathrm{en-ki}$ lugal 'abzu-a' [hé-pàd]

$11^{\prime}$ zi ddam-gal-'nun-na' [ hué-pàd]

$12^{\prime}$ zi drasal'-lú-'hni i [ ḩé-pàd ]

13' zi dpap-nun-[an-ki hué-pàd] 


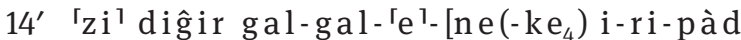
ha-ba-ra-du-un]

15' (Spuren; dann abgebrochen)

\section{Kommentar}

Rs. 3' Die Zeile wurde ergänzt nach BAM 8, 85 Taf. 2: 70; 99 Taf. 3: 46; 120 Taf. 3: 143; 197 Taf. 5: 105; 232 Taf. 6: 65; 417 Taf. 12: 102; 474 Taf. 13-15: 140; 489 Taf. 13-15: 227'; 532 Taf. 16: $174^{\prime}$.

Rs. 4' Die Zeile wurde ergänzt nach BAM 8, 85 Taf. 2: 71; 99 Taf. 3: 47; 120 Taf. 3: 144; 232 Taf. 6: 66; 474 ff. Taf. 13-15: 141, 180, 228', 265'; 532 Taf. 16: 175'.

Rs. $5^{\prime}$ Die Zeile wurde ergänzt nach BAM 8, 120 Taf. 3: 145; 474 Taf. 13-15: 142.

Rs. 6' Die geringfügigen in dieser Zeile erhaltenen Zeichen könnten zu der Lesung ${ }^{\mathrm{C}} \mathrm{me}$ h $\mathrm{ul}$ l-ĝál ${ }^{1}$ [ba r-šè h é - e m - ta - g ub] passen.

Rs. 7'-9' Zur Ergänzung der Zeilen 7'-9' siehe BAM 8, 194f. Taf. 5: 92-94 und 198f. Taf. 5: 113-115.

Rs. 14' Die Zeile wurde ergänzt nach BAM 8, 109 Taf. 3: 97; 153 Taf. 4: 116'; 227 Taf. 6: 37; 248 Taf. 6: 188'; vgl. 255 Taf. 7: 24; 286 Taf. 7: 167; 314 Taf. 9: 76'; 485 Taf. 13-15: 205.

\section{Literaturverzeichnis}

Abusch, T./D. Schwemer (2011): Corpus of Mesopotamian anti-witchcraft rituals, volume one. AMD 8. Leiden/Boston

Annus, A./A. Lenzi (2010): Ludlul bēl nēmeqi. The standard Babylonian poem of the righteous sufferer. SAACT 7. Helsinki

Attinger, P. (1993): Eléments de la linguistique sumérienne. La construction de $\mathrm{du}_{11} / \mathrm{e} / \mathrm{di}$ «dire», Fribourg Suisse/Göttingen

- (2008): La médecine mésopotamienne, JMC 11-12, 1-96

BAM 8 s. Geller (2016)

Borger, R. (21981): Assyrisch-babylonische Zeichenliste, 2. Auflage (Nachdruck der 1. Auflage mit Supplement 415-452). AOAT 33/33A. Kevelaer

- (22010): Mesopotamisches Zeichenlexikon. Zweite, revidierte und aktualisierte Auflage. AOAT 305. Münster

Brunke, H./W. Sallaberger (2010): Aromata für Duftöl, in: A.Kleinerman/J. M. Sasson (Hg.), Why should someone who knows something conceal it? Cuneiform studies in honor of David I. Owen on his 70th birthday, Bethesda

Casaburi, M. C. (2000): The alleged Mesopotamian «lent». The hemerology for tešritu, SEL 17, 13-29

Clay, A. T. (1923): Epics, hymns, omens, and other texts. BRM 4. New Haven

Couto, E. (2007): Conceptos de transmisión de la enfermedad en Mesopotamia. Algunas reflexiones, Historiae 4, 1-24

Dick, M. B. (1999): Born in heaven, made on earth: the making of the cult image in the ancient Near East. Winona Lake
Durand, J.-M. (1982): Documents cunéiformes de la IVe section de l'École Pratique des Hautes Études, 1. Catalogue et copies cunéiformes. Genève

- (2000): Documents épistolaires du palais de Mari, Tome 3. Paris

Ebeling, E. (1915): Assyrische Beschwörungen, ZDMG 69, 89-103

Falkenstein, A. (1931): Die Haupttypen der sumerischen Beschwörung literarisch untersucht. LSS NF 1. Leipzig

Farber, W. (1977): Beschwörungsrituale an Ištar und Dumuzi. Akademie der Wissenschaften und der Literatur, Veröffentlichungen der Orientalischen Kommission, Band 30. Wiesbaden

- (1987): Rituale und Beschwörungen in akkadischer Sprache, in: O. Kaiser (Hg.), Texte aus der Umwelt des Alten Testaments, Band 2: Religiöse Texte, Lieferung 2: Rituale und Beschwörungen in akkadischer Sprache. Gütersloh, 212-281

- (2004): How to marry a disease. Epidemics, contagion, and a magic ritual against the ,hand' of the ghost, in: H. F. J. Horstmanshoff/M. Stol (Hg.), Magic and rationality in ancient Near Eastern and Graeco-Roman medicine. Leiden/Boston, 117-132

Finkel, I. L. (2000): On Late Babylonian medical training, in: A. R. George/I. L. Finkel (Hg.), Wisdom, gods and literature. Studies in Assyriology in honour of W. G. Lambert. Winona Lake, 137-223

Geller, M J. (2010): Ancient Babylonian medicine. Theory and practice. Oxford

- (2016): Healing magic and evil demons. Canonical udug-hul incantations. BAM 8. Boston/Berlin

George, A. R. (1992): Babylonian topographical texts. Leuven Grayson. A. K. (1987): Assyrian rulers of the third and second millennia BC (to 1115 BC). RIMA 1. Toronto/London

Groneberg, B. (2006): Aspekte der „Göttlichkeit“ in Mesopotamien. Zur Klassifizierung von Göttern und Zwischenwesen, in: R. G. Kratz/H. Spieckermann (Hg.), Götterbilder, Gottesbilder, Weltbilder. Polytheismus und Monotheismus in der Welt der Antike, Band 1: Ägypten, Mesopotamien, Persien, Kleinasien, Syrien, Palästina. Tübingen, 131-166

Heeßel, N. P. (2000): Babylonisch-assyrische Diagnostik. AOAT 43. Münster

- (2006): The Babylonian physician Rabâ-ša-Marduk. Another look at physicians and exorcists in the ancient Near East, in: A. Attia/G. Buisson (Hg.), Advances in Mesopotamian medicine from Hammurabi to Hippocrates. Proceedings of the international conference „Oeil malade et mauvais oeil“, Collège de France, Paris, 23rd June 2006. CM 37. Leiden/Boston, 13-28

- (2012): Divinatorische Texte 2: Opferschau-Omina. KAL 5. Wiesbaden

Hrůša, I. (2010): Die akkadische Synonymenliste malku = šarru. Eine Textedition mit Übersetzung und Kommentar. Münster

Hunger, H. (1968): Babylonische und assyrische Kolophone. AOAT 2. Kevelaer

- (1989): MUL.APIN. An astronomical compendium in cuneiform. AfO Bh. 24. Horn

Jakob, S. (2003): Mittelassyrische Verwaltung und Sozialstruktur. Untersuchungen. CM 29. Leiden

Kinnier Wilson, J. (2005): Notes on the Assyrian pharmacological series uru.an.na : maštakal, JNES 64, 45-52

Knudtzon, J. A. (1915): Die El-Amarna-Tafeln, Band 1: Die Texte. Leipzig 
Krebernik, M. (1998): Nin-girima I. Beschwörungsgöttin, in: D. 0. Edzard (Hg.), RIA 9. Berlin/New York, 363-368

Labat, R. (1951): Traité akkadien de diagnostics et pronostics médicaux. Paris

- (61988): Manuel d'épigraphie akkadienne, 6. éd. augm. d'addenda par Florence Malbran-Labat. Paris

Laessøe, J. (1955): Studies on the Assyrian ritual and series bît rimki. Kopenhagen

Lambert, W. G. (2013): Babylonian creation myths. Winona Lake

Litke, R. L. (1998): A reconstruction of the Assyro-Babylonian god-lists, An : ${ }^{\mathrm{A}}$ A-nu-um and $\mathrm{AN}$ : Anu šá amēli. New Haven

Livingstone, A. (1986): Mystical and mythological explanatory works of Assyrian and Babylonian scholars. Oxford

- (1989): Court poetry and literary miscellanea. SAA 3. Helsinki

- (2014): Hemerologies of Assyrian and Babylonian scholars. CUSAS 25. Bethesda

Marzahn, J. (2008): Wahrheit. Eine Ausstellung des Vorderasiatischen Museums Staatliche Museen zu Berlin. München

- (2017): Ein Phantom-Griffel aus Assur - oder die Lust der Gestaltung, in: J. Gießauf (Hg.), Zwischen Karawane und Orientexpress. Streifzüge durch Jahrtausende orientalischer Geschichte und Kultur. Festschrift für Hannes Galter. AOAT 434. Münster, 195-209

Maul, S. M. (1994): Zukunftsbewältigung. Eine Untersuchung altorientalischen Denkens anhand der babylonisch-assyrischen Löserituale (Namburbi). BaF 18. Mainz am Rhein

- (2010): Die Tontafelbibliothek aus dem sogenannten „Haus des Beschwörungspriesters“, in: S. M. Maul/N. P. Heeßel (Hg.), Assur-Forschungen. Arbeiten aus der Forschungsstelle „Edition literarischer Keilschrifttexte aus Assur“ der Heidelberger Akademie der Wissenschaften. Wiesbaden, 189-228

Mayer, W. [R.] (1976): Untersuchungen zur Formensprache der babylonischen „Gebetsbeschwörungen“. StPohl SM 5. Rom

Meier, G. (1941-1944): Die zweite Tafel der Serie bīt mēseri, AfO 14, 139-152

Meinhold, W. (2009): Ištar in Aššur. Untersuchung eines Lokalkultes von ca. 2500 bis 614 v. Chr. AOAT 367. Münster

Meißner, B. (1915): Zu Ebeling’s Aufsatz ZDMG 69, 89 ff., ZDMG 69, 412-414

Menzel, B. (1981): Assyrische Tempel. StPohl SM 10. Rom

Miglus, P. (1996): Das Wohngebiet von Assur. Stratigraphie und Architektur. WVDOG 93. Berlin

Neufeld, E. (1986): The earliest document of contagious disease in Mesopotamia (Mari tablet ARM X, 129), JANER 18, 53-66
Oppenheim, A. L. (1956): The Interpretation of dreams in the ancient Near East. With a translation of an Assyrian dream-book. TAPS NS 46/3. Philadelphia

Oshima, T. (2014): Babylonian poems of pious sufferers. Ludlul bèl nēmeqi and the Babylonian theodicy. Orientalische Religionen in der Antike 14. Tübingen

Parpola, S. (1983): Letters from Assyrian scholars to the kings Esarhaddon and Assurbanipal, 2. commentary and appendices. AOAT 5/2. Kevelaer

- (1993): Letters from Assyrian and Babylonian scholars. SAA 10. Helsinki

Pedersén, O. (1986): Archives and libraries in the city of Assur: a survey of the material from the German excavations, Part 2. Uppsala

Reiner, E. (1956): lipšur-litanies, JNES 15, 129-149

- (1995): Astral magic in Babylonia. TAPS 85/4. Philadelphia

Scheil, V. (1921): Catalogue de la Collection Eugène Tisserant, RA 18, 1-33

Schwemer, D. (2017): The anti-witchcraft ritual Maqlû. The cuneiform sources of a magic ceremony from ancient Mesopotamia, Wiesbaden

Schuster, A. (2008): Steine als Schutz- und Heilmittel. Untersuchung zu ihrer Verwendung in der Beschwörungskunst Mesopotamiens im 1. Jt. v. Chr. AOAT 46. Münster

Scurlock, J. (2006): Magico-medical means of treating ghostinduced illnesses in ancient Mesopotamia. AMD 3. Leiden

Soden, W. von (1964): Die Schutzgenien Lamassu und Schedu in der babylonisch-assyrischen Literatur, BaM 3, 148-156

Soden W. von/W. Röllig (31976): Das akkadische Syllabar. 3. Auflage durchgesehen und verbessert. AnOr 42. Rom

Streck, M. P. (1998): Ninurta/Ninĝirsu. a. I. In Mesopotamien, in: D. O. Edzard (Hg.), RlA 9. Berlin/New York, 512-522

Stol, M. (1993): Epilepsy in Babylonia. CM 2. Groningen

- (1998): Einige kurze Wortstudien, in: S. M. Maul (Hg.), Festschrift für Rykle Borger zu seinem 65. Geburtstag am 24. Mai 1994. tikip santakki mala bašmu.... CM 10. Groningen, 343-352

Stol, M. (2000): Birth in Mesopotamia and the Bible. Its Mediterranean setting. Groningen

Thompson, R. C. (1924): A Babylonian explanatory text, JRAS 56, 452-457

- (1949): A dictionary of Assyrian botany. London

Vorländer, H. (1975): Mein Gott. Die Vorstellungen vom persönlichen Gott im Alten Orient und im Alten Testament. AOAT 23. Kevelaer van Wyk, B.-E. [u. a.] (2004): Handbuch der Arzneipflanzen. Ein illustrierter Leitfaden. Stuttgart 